\title{
A p53/TIAF1/WWOX triad exerts cancer suppression but may cause brain protein aggregation due to p53/WWOX functional antagonism
}

Pei-Yi Chou', Sing-Ru Lin' ${ }^{1}$ Ming-Hui Lee ${ }^{1}$, Lori Schultz ${ }^{2}$, Chun-I Sze ${ }^{3}$ and Nan-Shan Chang ${ }^{1,2,4,5^{*}}$ (D)

\begin{abstract}
Background: Tumor suppressor WWOX physically binds p53 and TIAF1 and together induces apoptosis and tumor suppression. To understand the molecular action, here we investigated the formation of WWOX/TIAF1/p53 triad and its regulation of cancer cell migration, anchorage-independent growth, SMAD promoter activation, apoptosis, and potential role in neurodegeneration.
\end{abstract}

Methods: Time-lapse microscopy was used to measure the extent of cell migration. Protein/protein interactions were determined by co-immunoprecipitation, FRET microscopy, and yeast two-hybrid analysis. The WWOX/TIAF1/p53 triadmediated cancer suppression was determined by measuring the extent of cell migration, anchorage-independent growth, SMAD promoter activation, and apoptosis. p53-deficient lung cancer cell growth in nude mice was carried out to assess the tumor suppressor function of ectopic p53 and/or WWOX.

Results: Wwox-deficient MEF cells exhibited constitutive Smad3 and p38 activation and migrated individually and much faster than wild type cells. TGF- $\beta$ increased the migration of wild type MEF cells, but significantly suppressed Wwox knockout cell migration. While each of the triad proteins is responsive to TGF- $\beta$ stimulation, ectopically expressed triad proteins suppressed cancer cell migration, anchorage-independent growth, and SMAD promoter activation, as well as caused apoptosis. The effects are due in part to TIAF1 polymerization and its retention of p53 and WWOX in the cytoplasm. p53 and TIAF1 were effective in suppressing anchorage-independent growth, and WWOX ineffective. p53 and TIAF1 blocked WWOX or Smad4-regulated SMAD promoter activation. WWOX suppressed lung cancer NCI-H1299 growth and inhibited splenomegaly by inflammatory immune response, and p53 blocked the event in nude mice. The p53/MWOX-cancer mice exhibited BACE upregulation, APP degradation, tau tangle formation, and amyloid $\beta$ generation in the brain and lung.

Conclusion: The WWOX/TIAF1/p53 triad is potent in cancer suppression by blocking cancer cell migration, anchorageindependent growth and SMAD promoter activation, and causing apoptosis. Yet, p53 may functionally antagonize with WWOX. p53 blocks WWOX inhibition of inflammatory immune response induced by cancer, and this leads to protein aggregation in the brain as seen in the Alzheimer's disease and other neurodegeneration.

Keywords: Tumor suppressor, WWOX, p53, TIAF1, Cell migration, Promoter activation

\footnotetext{
* Correspondence: changns@mail.ncku.edu.tw

'Institute of Molecular Medicine, National Cheng Kung University, College of

Medicine, Tainan, Taiwan 70101, Republic of China

${ }^{2}$ Laboratory of Molecular Immunology, Guthrie Research Institute, Sayre, PA

18840, USA

Full list of author information is available at the end of the article
}

(c) The Author(s). 2019 Open Access This article is distributed under the terms of the Creative Commons Attribution 4.0 International License (http://creativecommons.org/licenses/by/4.0/), which permits unrestricted use, distribution, and reproduction in any medium, provided you give appropriate credit to the original author(s) and the source, provide a link to the Creative Commons license, and indicate if changes were made. The Creative Commons Public Domain Dedication waiver (http://creativecommons.org/publicdomain/zero/1.0/) applies to the data made available in this article, unless otherwise stated. 


\section{Background}

WW domain-containing oxidoreductase, designated WWOX, FOR or WOX1, is a 46-kDa tumor suppressor protein ([1-6]; reviews). WWOX gene is located on a common fragile site FRA16D on chromosome ch16q23.3-24.1, encompassing a million bases [1-8]. Loss of WWOX protein occurs as a result of genetic alterations [1-6], promoter hypermethylation [9-12], and translational blockade [13], and this may be associated with cancer development [1, 2, 4-6]. Significant downregulation of WWOX protein is frequently seen in metastatic cancer cells [1-6]. Loss of WWOX upregulates the JAK2/STAT3 pathway that drives cancer metastasis in triple negative breast cancer cells [14]. Also, loss of WWOX in ovarian cancer cells acquires enhanced migration and metastasis due to altered interactions between integrin $\alpha 3$ and fibronectin [15]. WWOX suppresses the expression of RUNX2 and thereby blocks the invasion and metastasis of osteosarcoma and lung cancer cells $[16,17]$.

Despite its connection with cancer, WWOX indeed plays a critical role in neural development and neurodegeneration. $W W O X$ gene has recently been determined as a risk factor for Alzheimer's disease [3, 18]. Null mutations of $W W O X / W w o x$ gene cause severe neural diseases (e.g. epilepsy, microcephaly, retinal degeneration, and ataxia), metabolic disorders (including lipid, cholesterol and glucose metabolism), and early death in the newborns $[1,3,19,20]$. No spontaneous tumor growth is shown in the newborns of humans and rats, suggesting that WWOX primarily maintains the physiology of normal tissues and organs. WWOX participates in the neural development $[3,21]$. WWOX deficiency leads to rapid protein aggregation to cause neuronal damage and death in vivo [3, 22-26]. For example, shortly after birth for 15 days, Wwox knockout mice develop brain protein aggregation, including TRAPPC6A $\triangle$ (Trafficking protein particle complex 6A delta) [24-27], TIAF1 (TGFß1-Induced Anti-Apoptotic Factor 1) [23, 24, 28], SH3GLB2 (SH3 Domain Containing GRB2 Like, Endophilin B2) [26], tau $[3,26]$ and amyloid $\beta[3,26]$, become aggregated in the brains of newborn [22-28]. Loss of WWOX probably induces conformational changes of the aforementioned proteins leading to aggregation.

Transiently overexpressed WWOX with Tyr33 phosphorylation (pY33-WWOX) induces apoptosis $[1,3,6$, 29-37]. pY33-WWOX also maintains the normal physiology of cells $[1,3,6]$. pY33-WWOX works together with p53, Hyal-2 and Smad4 to induce apoptosis [21-23, $34,35]$. In response to UV and cold shock, WWOX, p53 and NOS2 (nitric oxide synthase 2) generate a novel type of cell death, termed bubbling cell death, in many types of cells [35-37]. When overexpressed, ectopic Hyal-2/WWOX/Smad4 signaling complex causes bubbling cell death in response to high-molecular-weight hyaluronan of 2-4 million Daltons [35, 37]. In contrast, hyaluronan increases the binding and signaling of $\mathrm{p} 53$ / WWOX/Smad4 for leading to membrane blebbing, but without causing cell death [35, 37].

During the early stage of cancer development, WWOX is upregulated in the hyperplasia tissues [13, 38]. Activated pY33-WWOX is rapidly upregulated in $24 \mathrm{~h}$ during the acute phase of UVB irradiation-induced skin squamous cell carcinoma (SCC) in hairless mice [13]. pY33-WWOX probably exerts its tumor suppressor function to limit cancer progression. pY33-WWOX disappears in 3 months in the UVB-treated mice as SCC continuously develops, even though the mRNA coding for Wwox is still present. Similarly, pY33-WWOX upregulation is observed during breast cancer progression to a premetastatic state [38]. Estrogen induces the upregulation and activation of WWOX [38]. Perhaps, upregulated pY33-WWOX can be regarded as a marker for the very early stage of cancer progression.

We have reported the protein complex of WWOX, p53 and TIAF1 triad is a potential axis of tumor suppression $[23,28]$. Here, we examined whether the protein triad suppresses anchorage-independent growth, blocks cell migration, inhibits SMAD promoter activation, and causes apoptosis. We determined the role of p53 phosphorylation at Ser46 in contributing to the apoptotic function of the triad. The kinetics of the triad formation was examined by FRET (Förster resonance energy transfer) microscopy, co-immunoprecipitation, and yeast two-hybrid analysis [22-26, 28-32, 34-39]. Without activation, p53 cannot bind TIAF1 [40]. Intriguingly, activated p53 binds TIAF1 and then together with WWOX to form a stabilized triad. Among p53 isoforms, $\triangle 133 \mathrm{p} 53 \gamma$ is most potent in suppressing the migration of WWOX-negative MDAMB-231 cells, which correlates with its activation of SMAD promoter. Notably, p53 may functionally antagonize with WWOX. p53 blocks WWOX inhibition of inflammatory immune response induced by cancer, and this leads to protein aggregation in the brain as seen in the Alzheimer's disease $(\mathrm{AD})$ and other neurodegeneration.

\section{Methods}

\section{Cell lines and cell culture}

Cell lines used in this study included human breast MCF-7 and MDA-MB-231 cancer cells [38], human prostate DU145 cells [35], human monocytic U937 cells [35], human lung p53-deficient NCI-H1299 [35], murine L929 fibroblasts [29-31], monkey kidney SV40 virustransformed COS7 fibroblasts [31, 35], and primary mink lung epithelial Mv1Lu cells [40, 41] (American Type Culture Collection). Mouse embryonic fibroblast (MEF) for Wwox wild type, heterozygous and knockout cells were generated and maintained in RPMI-1640 medium supplemented with $10 \%$ fetal bovine serum 
[25]. All the cells were cultured at $37^{\circ} \mathrm{C}$ in an incubator with $5 \% \mathrm{CO}_{2} /$ atmosphere.

\section{Chemicals, antibodies, Western blotting, immunofluorescence microscopy, and co-immunoprecipitation}

Recombinant TNF $\alpha$, TGF- $\beta 1$ and $-\beta 2$ proteins were from PeproTech. Phalloidin for F-actin staining was from Invitrogen. Antibodies against ERK (extracellular signalregulated kinase), pERK (phosphorylated ERK), BACE ( $\beta$-secretase), WWOX, p53, BECN-1 (Beclin-1), IкB $\alpha$ (inhibitor of nuclear factor kappa B alpha), Foxp3 (Forkhead box P3), AIF (apoptosis inducing factor) and GFP (green fluorescent protein) were from Santa Cruz Biotechnology. Additional commercial antibodies used were against: A $\beta$ (AbD Serotec), APP (EMD Millipore), NFT (neurofibrillary tangles; Invitrogen), and $\alpha$-tubulin (Sigma-Aldrich) [21-25]. Homemade antibodies against WWOX were also used [13, 21-25]. Antibodies were used for Western blotting analysis and immunofluorescent microscopy, as described [21-25, 29-31]. Where indicated, binding of WWOX with TIAF1 in response to TNF $\alpha$ and TGF- $\beta 1$ were determined by co-immunoprecipitation using specific antibodies, as described [29-31, 41].

\section{CDNA constructs and electroporation}

We have first isolated the murine full-length Wwox cDNA [29]. Full-length dominant-negative Wwox construct was mutated on Lys28 to Thr28 and Asp29 to Val29 in the first WW domain (dnWwox) [30]. Another dominant negative construct contained the $N$-terminal WW domains with the same mutation $(\mathrm{dn} w w)$ [30]. A wild type TIAF1 [40-42] and 3 TIAF1 dominant negative constructs were made: TIAF1 (S6G) and TIAF (S37G) with Ser6 mutated to glycine and Ser37 to glycine, respectively, and TIAF1 (S68A) with Ser68 mutated to alanine. Ser37 is a confirmed phosphorylated site [22-24]. These three mutation sites were the predicted phosphorylation sites in TIAF1 protein by using NetPhos 2.0 Server (Technical University of Denmark). Other constructs used were p53 and p53(S46G) [23, 29-31], and eight p53 isoforms [43-46]. p53 isoforms were kind gifts of Dr. JC Bourdon of the University of Dundee. All of the constructs used were made in pEGFP-C1, pECFP-C1 and pDsRed vectors (Clontech), respectively. Wwox siRNA (Wwoxsi) was designed and cloned into pSuppressorNeo vector (Imgenex) [47]. Designed primers for WWOXsi\#1 and \#2 siRNAs, targeting a common DNA sequence in human/murine WWOX and another DNA sequence in human WWOX, respectively, were made [47]. Cells were electroporated twice with the indicated DNA constructs $(200 \mathrm{~V}, 50 \mathrm{~ms})$ and cultured in medium containing $10 \%$ FBS overnight prior to carrying out experiments. While indicated, liposome-based GeneFECTOR (Venn Nova) was used to transfect cells with the expression constructs.
Cell migration assay, promoter activation, and time-lapse microscopy

Cell migration assay was performed as described [24]. A culture insert (ibidi) was placed onto a $35 \mathrm{~mm}$ dish, and an equal number of cells $\left(70 \mu \mathrm{l}, 4 \times 10^{5}\right.$ cells) were seeded into the two reservoirs of the same insert, so there would be a $500 \pm 50 \mathrm{~mm}$ gap. After overnight incubation at $37^{\circ} \mathrm{C} / 5 \% \mathrm{CO} 2$, the insert was gently removed and the medium was changed to serum-free, or contain $2 \%$ FBS to minimize cell proliferation. The cell migration was imaged at an indicated time interval for 24 to $48 \mathrm{~h}$ using a NIKON TE2000-U microscope [23-26]. Cell migration was analyzed either by counting the migrating cell numbers or by measuring the migrating cell areas. An inverted Olympus IX81 fluorescence microscope was used for carrying out time-lapse microscopy [34-37]. Cell migration rate was measured by cell migrating distance versus time. Single cell moving path was tracked using the $\mathrm{NIH}$ Image $J$ manual tracking and chemotaxis and migration tool.

\section{Tri-molecular FRET microscopy}

The kinetics of tri-molecular protein/protein binding interactions was carried out by FRET microscopy [35, 37, 39]. Experiments were designed to let the FRET energy transfer from WWOX-ECFP to TIAF1-EGFP, and finally to p53DsRed. COS7 cells were transiently overexpressed with ECFP-WWOX, EGFP-TIAF1, and DsRed-p53. In negative controls, cells were transfected with ECFP, EGFP, and DsRed. Following culturing overnight, cells were treated with Prima-1 $(10 \mu \mathrm{M})$, an activator of p53 activator, for 0 , 30 and $60 \mathrm{~min}$, followed by fixing with $4 \%$ paraformaldehyde. Similar experiments were carried out by treating cells with TGF- $\beta 1 \quad(10 \mathrm{ng} / \mathrm{ml})$. FRET microscopy was performed using an inverted fluorescence microscope (Nikon Eclipse TE-2000 U), and data analyzed as described $[35,37,39]$. The FRET images were corrected for background fluorescence from an area free of cells and spectral bleed-through. The spectrally corrected FRET concentration (FRETc) was calculated by Youvan's equation (using a software program Image-Pro 6.1, Media Cybernetics): FRETc $=[$ fret - bk $($ fret $)]-c f($ don $) \times[$ don $-b k($ don $)]$ $-\mathrm{cf} .(\mathrm{acc}) \times[\mathrm{acc}-\mathrm{bk}(\mathrm{acc})]$, where fret $=$ fret image, $\mathrm{bk}=$ background, $\mathrm{cf} .=$ correction factor, don $=$ donor image, and acc $=$ acceptor image. The equation normalizes the FRET signals to the expression levels of the fluorescent proteins.

\section{Cytoplasm-based yeast two-hybrid analysis for protein/ protein binding interactions}

To investigate whether WWOX binds TIAF1 in vivo, Ras rescue-based yeast two-hybrid analysis (CytoTrap; Stratagene) was performed [29-31, 34, 35]. In brief, binding of a cytosolic Sos-tagged bait protein to a cell membraneanchored target protein (tagged with a myristoylation 
signal) leads to activation of the Ras signaling pathway in yeast. This activation allows mutant yeast cdc $25 \mathrm{H}$ to grow in $37^{\circ} \mathrm{C}$ using a selective agarose plate containing galactose. Without binding, yeast cells fail to grow at $37^{\circ} \mathrm{C}$. Target constructs made in a pMyr vector (with the myristoylation signal) were murine TIAF1 and human p53. Bait constructs made in a pSos vector (tagged with an $\mathrm{N}$-terminal Sos protein) were murine WWOX, WWOXww (the first WW domain), WWOX(Y33R), and WWOXsdr (the entire SDR domain). Additionally, self-binding of MafB (in both pMyr and pSos) was regarded as a positive control, and empty pSos versus empty pMyr as a negative control.

\section{Cell cycle analysis}

Cells were electroporated with indicated EGFP-tagged plasmids and cultured overnight. The electroporation efficiency was confirmed by fluorescence microscopy. The condition media were then harvested, and the cells collected by trypsin and centrifugation ( $4000 \mathrm{rpm}, 10 \mathrm{~min}$ ) . Cells were washed once with PBS and then were fixed in $70 \%$ ethanol overnight. After overnight fixation, cells were then washed once with PBS and stained with propidium iodide (PI) solution $(2 \mu \mathrm{g} / \mathrm{ml} \mathrm{PI}, 10 \mu \mathrm{g} / \mathrm{ml}$ RNase A in PBS) for $30 \mathrm{~min}$ at room temperature. Cell cycle analysis was performed by flow cytometry (BD) [23, 24, 29-31].

\section{Cell proliferation assay}

$5 \times 10^{4}$ cells per well were seeded in 12-well microtiter plates, cultured overnight in medium containing $10 \%$ FBS, and then trypsinized. The cell number was counted using hemocytometer at time point $0,6,18,24$, and $48 \mathrm{~h}$.

\section{Soft agarose colony survival assay}

Adherence-independent cell growth or transforming growth was performed in a soft agarose colony survival assay [40]. MDA-MB-231 or indicated cells were electroporated with expression constructs of p53-DsRed, WWOXECFP, and/or EGFP-TIAF1. These cells were plated at a density of $3 \times 10^{4}$ cells/35-mm dish in triplicate in RPMI $1640,10 \%$ fetal bovine serum, $0.8 \%$ agarose, and $10 \mathrm{mM}$ HEPES. Dishes were incubated in a humidified $\mathrm{CO} 2$ incubator at $37^{\circ} \mathrm{C}$ for 3 weeks. Live colonies were stained with the MTS proliferation reagent (Promega) and counted. In controls, cells were treated with DMEM medium or subjected to electroporation with medium only.

\section{Cancer cell growth in nude mice}

p53-deficient NCI-H1299 lung cancer cells were transiently overexpressed with p53-DsRed and/or WWOXCFP, or EGFP only using liposome-based GeneFECTOR (Venn Nova). Nude mice received subcutaneous injections of these cells twice on both sides of the flanks, followed by measuring the tumor sizes daily, as described [26].

\section{Statistical analysis}

Data were analyzed by Student's $t$ test among controls and tested groups using Microsoft excel. Data were expressed as mean \pm standard deviation, where $p<0.05$ was considered significant.

\section{Results}

\section{Altered signaling in Wwox knockout MEF cells}

Wwox gene knockout mice and wild type $W w o x^{+/+}$, knockout $W w o x^{-/-}$, and heterozygous $W w o x^{+/-}$mouse embryonic fibroblasts (MEF) were established [25]. Wild type MEF cells tightly merge with each other and appear squamous (and roundish for dividing cells) (Fig. 1a). Wwox knockout MEF cells appear similarly to that of the wild type cells, and have loose intercellular connections (Fig. 1a). Compared to the heterozygous Wwox MEF cells, knockout cells have a significant reduction in expression of endogenous p53, IкB $\alpha$ and Fas (Fig. 1b). TNF $\alpha$ did not restore the protein levels during treatment of knockout cells for $1 \mathrm{~h}$ (Fig. 1b). Interestingly, knockout cells have a higher expression of p63 than the heterozygous cells (Fig. 1b). The protein levels for p53, p63 and WWOX in the wild type cells are shown (Fig. 1c). Smad3 of the SMAD pathway was constitutively phosphorylated (or activated) in the knockout cells (Fig. 1d). Also, in the knockout cells, inhibitor Smad6 was significantly downregulated (Fig. 1d). Smad2, 3 and 6 were responsive to TNF $\alpha$-mediated upregulation in the heterozygous cells, but not in the knockout cells (Fig. 1d). In the MAPK pathway, p38 is constitutively activated in the knockout cells (Fig. 1e). TNF $\alpha$ induced the activation of JNK1 in both heterozygous and knockout cells (Fig. 1e). Together, compared to the heterozygous cells, knockout MEF cells exhibit many aberrant signaling pathways.

\section{WWOX is a potent inhibitor of cell migration}

Cell proliferation assay was performed to examine the doubling time of wild type and Wwox knockout MEF cells. Under normal culture condition (medium containing $10 \% \mathrm{FBS}$ ), the proliferation rate of wild type MEF cells was significantly slower than the knockout MEF cells (Fig. 2a and b). Both Wwox knockout and heterozygous MEF cells migrated significantly faster than the wild type cells (Fig. 2c and d). Wild type cells migrated collectively, while WWOX-deficient cells migrated individually (Fig. 2d and Additional file 1: Figure S1 for the enlarged image; Additional file 2: Video S1 and Additional file 3: Video S2).

Metastatic breast cancer MDA-MB-231 cells were used in the cell migration assay. MDA-MB-231 is a triple negative breast cancer cell line, which lacks the expression of estrogen receptor (ER), progesterone receptor (PR) and HER-2, and has a very low level of WWOX due to promoter hypermethylation. MDA-MB-231 cells migrate 


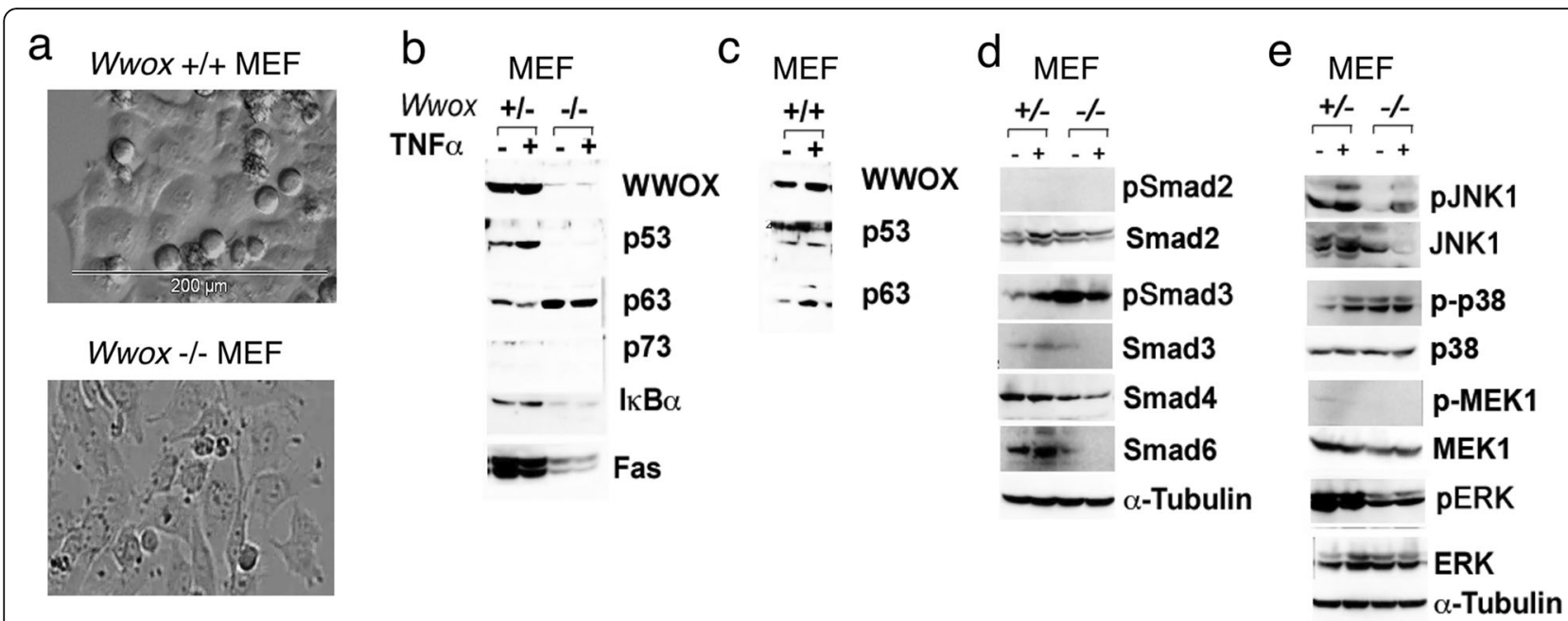

Fig. 1 Altered signaling in Wwox knockout MEF cells. a Wild type MEF Wwox ${ }^{+/+}$cells tightly merge with each other and appear squamous. Dividing cells at metaphase are roundish. Similar observations were shown for heterozygous Wwox ${ }^{+-}$MEF cells. Knockout Wwox ${ }^{-1-}$ MEF cells exhibit loose cell-cell contacts. b, c Wwox MEF cells were treated with TNFa for $1 \mathrm{~h}$. Protein expression in the TNF pathway is shown. $\mathbf{d}$ Under similar conditions, protein expression in the SMAD pathway is shown. Smad3 is constitutively phosphorylated or activated in the knockout cells. e Expression of proteins in the MAPK pathway is shown. P38 is constitutively activated

individually. In contrast, $\mathrm{ER}^{+}$and $\mathrm{WWOX}^{+}$breast MCF-7 cells migrate collectively. Transiently overexpressed WWOX suppressed the migration of MDAMB-231 cells, whereas inhibition of WWOX by dominant negatives (K28 T/D29V mutation in the full length WWOX or WW domains only) [30, 31], or by small interfering RNA (WWOXsi) [47], significantly enhanced the cell migration (Fig. $2 \mathrm{~d}$ and e). Similarly, we examined the migration of WWOX-negative MDA-MB-435 s and WWOX-positive L929 (or L929s) fibroblasts and breast MCF7 cancer cells. Without WWOX, cells migrated faster than those of WWWOX positive cells (Additional file 1: Figure S2). WWOX appears to be functionally deficient in MCF7 cells, as these cells effectively migrated similarly to the WWOX-negative MDA-MB-435 s and MDA-MB-231 cells (Additional file 1: Figure S2). L929 s cells are sensitive to tumor necrosis factor (TNF)-mediated apoptosis [29].

\section{TGF- $\beta 1$ enhances migration of $W w_{0 x}{ }^{+/+}$MEF cells but} suppresses that of $W_{w o x}{ }^{-1-}$ MEF cells

Transforming growth factor- $\beta 1$ (TGF- $\beta 1$ ) has both tumor suppressive and tumor promoting functions [48]. In the early stage of tumor progression, TGF- $\beta$ acts as a tumor suppressor by inducing apoptosis and inhibiting tumor cell growth, due in part to its activation of the proapoptotic WWWOX and Smad4 [33, 34]. In the late stage, metastatic tumor cells have altered TGF- $\beta$ receptors or Smads in the TGF- $\beta$ pathway, thereby resulting in TGF- $\beta$ mediated cancer growth. TGF- $\beta$ induces WWOX activation via Tyr33 phosphorylation and nuclear translocation to suppress cancer growth, and that loss of WWOX is found in a majority of metastatic cancer cells $[6,34]$.
Here, we investigated whether TGF- $\beta$ affects the cell migration of wild type and Wwox knockout MEF cells. TGF- $\beta 1$ marginally promoted the wild type MEF cell migration (Fig. 2g). However, TGF- $\beta 1$ suppressed Wwox knockout MEF cells migration (Fig. 2h). At $10 \mathrm{ng} / \mathrm{ml}$, TGF- $\beta 1$ significantly enhanced the migration of wild type cells, but not Wwox knockout cells (Fig. 2g, h). TGF- $\beta 2$ was more effective in suppressing the migration of Wwox knockout cells than TGF- $\beta 1$ (Fig. 2i and Additional file 1: Figure S3). TFG- $\beta 1$ had no significant effect on the cell proliferation in both wild type and the Wwox knockout MEF cells (Additional file 1: Figure S4).

\section{The TIAF1/WWOX/p53 axis inhibits cell migration}

We have reported the presence of the TGF- $\beta$-responsive TIAF1/WWOX/p53 complex as a molecular triad in tumor suppression [23, 40-42]. Suppression of TIAF1 by siRNA enhances cancer cell growth and abolishes WWOX-mediated apoptosis [23]. Similarly, p53-mediated apoptosis is blocked by siRNA targeting TIAF1 or WWOX [23]. p53 plays an inhibitory role in cell motility. p53 blocks epithelial-mesenchymal transition (EMT) and cell migration to prevent metastasis $[48,49]$. Loss of p53 expression in MEF cells leads to amoeboid-like movement and increased invasive ability $[50,51]$. WWOX physically binds p53 [23, 29-31], whereas p53 does not bind TIAF1 [42], suggesting that WWOX is a bridge for the formation of the TIAF1/WWOX/p53 triad to exert tumor suppression. TIAF1 binds and retains Smad2/3/4 in the cytoplasm and blocks Smad-mediated transcriptional activation [23].

To determine whether the protein triad controls cell migration, human breast MDA-MB-231 and MCF-7 cells 

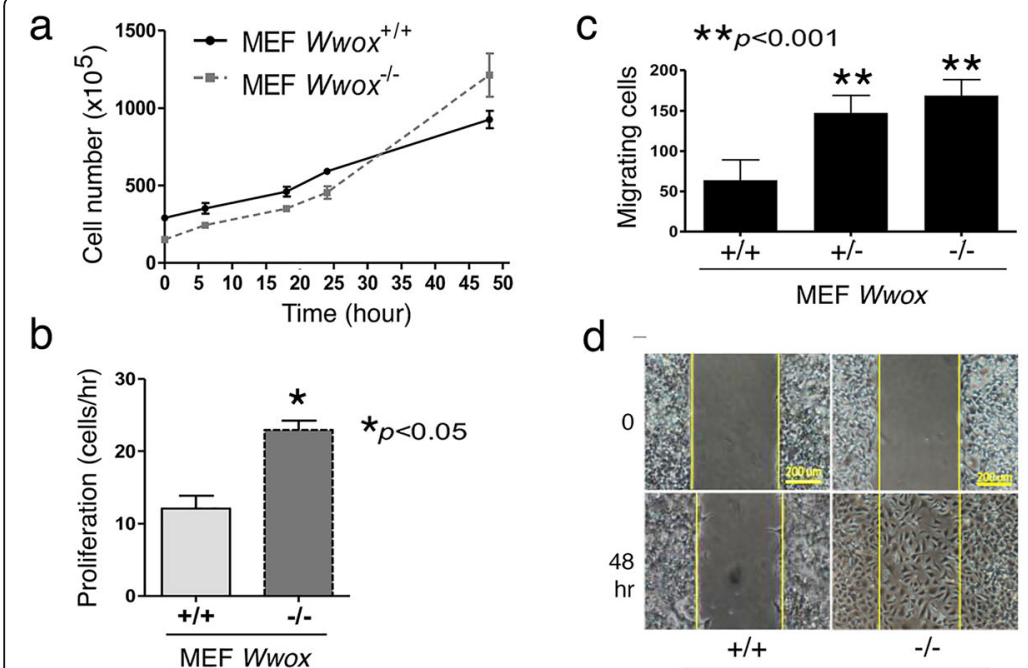

$\mathrm{d}$

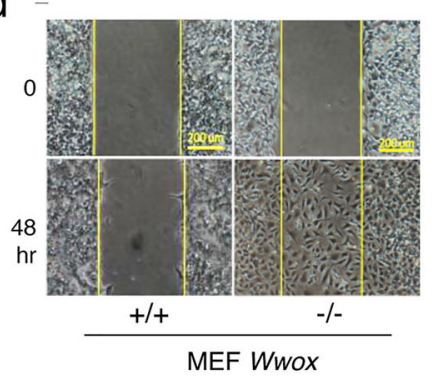

f
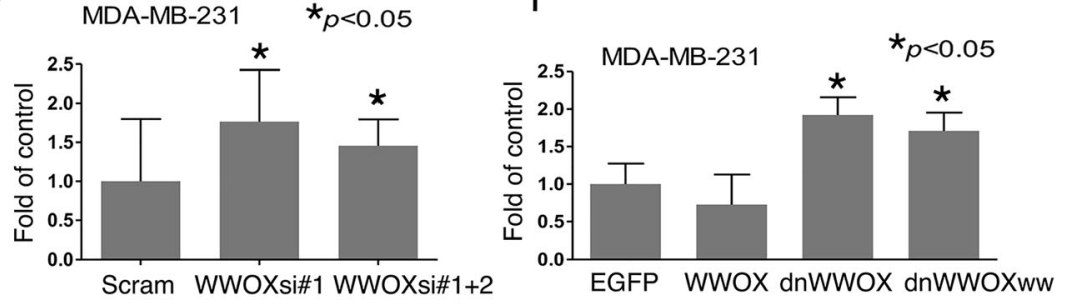

g
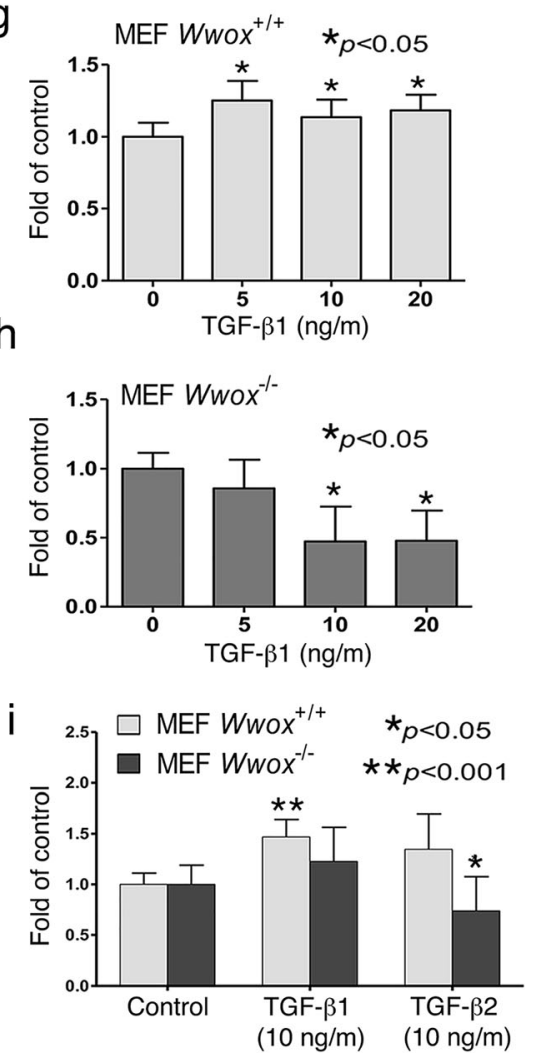

Fig. 2 WWOX inhibits cell proliferation and migration. a MEF cells were seeded on a 24-well plate and cultured for indicated periods. Live cell numbers were counted using a hemocytometer. $\mathbf{b}$ Wwox knockout MEF cells had a higher proliferation rate than wild type cells during culturing for $48 \mathrm{~h}$ (Mean \pm S.D., $n=3$, Student's $t$ test, $p<0.05$ ). $\mathbf{c ~ W w o x}{ }^{-/-}$and Wwox ${ }^{+/-}$MEF cells migrated significantly faster than wild type Wwox ${ }^{+/+}$cells ( $n=3$, Student's $t$ test). $\mathbf{d}$ Wild type cells migrated collectively, while WWOX-deficient cells migrated individually (see Additional file 2: Video S1 and Additional file 3: Video S2). e, f Breast MDA-MB-231 cancer cells were transfected with the indicated constructs by electroporation. Transiently overexpressed WWOX suppressed cell migration, whereas dnWWOX and WWOX siRNA constructs enhanced the migration. Dn: dominant negative; WWOXww: WW domain of WWOX; Scram: scramble control; WWOXsi\#1 and \#2: siRNAs targeting human/murine WWOX ( $n=3$, Student's $t$ test) [47]. $\mathbf{g}, \mathbf{h}$ During treatment for 48 h, TGF- $\beta 1$ promoted wild type cell migration, but suppressed Wwox knockout cell migration in a dose-dependent manner ( $n=3$, Student's $t$ test). i. Both TGF- $\beta 1$ and TGF- $\beta 2$ promoted wild type MEF cell migration during treatment for $48 \mathrm{~h}$. TGF- $\beta 2$ is more effective in suppressing Wwox knockout cell migration than TGF- $\beta 1(n=3$, Student's $t$ test $)$

were transiently overexpressed with the cDNA expression constructs for WWOX, TIAF1, and/or p53. Protein expression was examined by a fluorescent microscope. More than $60 \%$ of cells were positive for protein expression. Ectopic WWOX, TIAF1, and p53 significantly suppressed the migration of WWOX-deficient MDA-MB231 and WWOX-positive MCF-7 cells (Fig. 3a and b). A similar extent of migration inhibition was observed by using pairs of expression constructs, including WWOX/ p53, WWOX/TIAF1, and TIAF1/p53 (Fig. 3a and b). This is in agreement with our previous observation that ectopic TIAF1/WWOX/p53 triad suppresses the migration of L929 cells [23]. TGF- $\beta$, TNF- $\alpha$, apoptotic stress, or protein overexpression causes TIAF1 undergoes self-aggregation and the aggregates retain Smad proteins to prevent nuclear translocation [22, 23, 28]. Transiently overexpressed TIAF1 underwent aggregation and recruited p53 and WWOX in the aggregates in the cytoplasm (see punctate; Fig. 3c and S5 for enlarged image and additional data), suggesting a likely mechanism for migration inhibition.

\section{p53 is more potent than WWOX in suppressing anchorage-independent cell growth}

In addition, WWOX, TIAF1 and p53, together or in various combinations, inhibited anchorage-independent growth (or transforming growth) of MDA-MB-231 cells, as determined by soft agarose assay (Fig. 3d and e). Compared to p53 and TIAF1, WWOX was much less effective or ineffective in inhibiting the colony formation. Similarly, by using L929 cells, p53 is more potent than WWOX in blocking anchorage-independent growth of L929 cells (Additional file 1: Figure S6). p53 $\Delta S 46$ has a reduced apoptotic activity and fails to bind WWOX [31], whereas it was potent in blocking the colony formation (Additional file 1: Figure S6). We have previously 


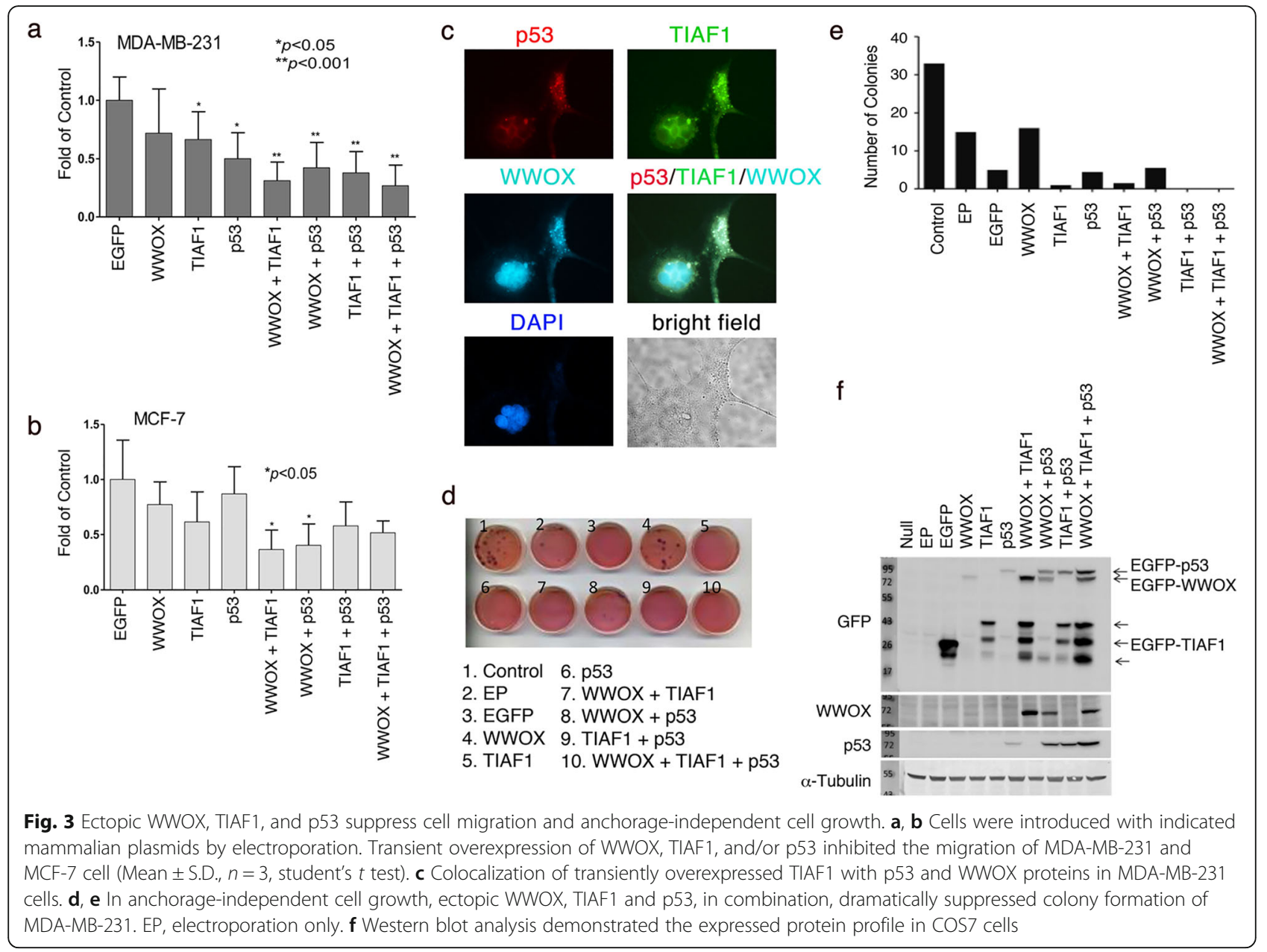

reported that TIAF1 and p53 are equally potent in suppressing anchorage-independent growth of L929 cells [40].

To further confirm the expression of ectopic proteins, COS7 cells were introduced with GFP-tagged WWOX, TIAF1, and p53, respectively, or in combinations (Fig. 3f). The GFP antibody probed the GFP-tagged proteins, and showed the ectopic protein expressions in COS7 cells. Compared with WWOX expression alone, co-expression of ectopic EGFP-tagged WWOX, TIAF1 and/or p53 upregulated the endogenous WWOX protein expression. Similarly, endogenous p53 was upregulated by ectopic expression of EGFP-tagged p53, WWOX, and/or TIAF1 (Fig. 3f). The reason for using COS7 cells is that this cell line is easy to transfect and ectopic proteins can be readily overexpressed in the cells [29-31].

\section{Ectopic $\Delta 133 p 53 \gamma$ activates SMAD promoter, which} correlates with suppression of cancer cell migration

Next, we investigated whether p53 and isoforms control cell migration. p53 has at least 12 isoforms [43-46]. Abnormal expression of p53 isoforms has been reported in several human cancers, such as head and neck cancer and ovarian tumors [42-45], indicating that p53 isoforms participate in tumor progression. MDA-MB-231 cells were transfected with wild type p53 and isoform cDNA expression constructs by electroporation (Fig. 4a). The results showed that many p53 isoforms decreased MDAMB-231cell migration, and that $\Delta 133 \mathrm{p} 53 \gamma$ was most effective (Fig. 4b). We have determined that when cells undergo over-activation of the SMAD promoter, apoptosis occurs [34]. Ectopic $\Delta 133 \mathrm{p} 53 \gamma$ caused the SMAD promoter activation (Fig. 4c and d). In contrast, wild type 53 and $\Delta 133$ p53 had no effect. TIAF1 binds Smad4 and blocks the SMAD responsive element from activation [23]. Knockdown of TIAF1 by siRNA did not increase $\Delta 133 \mathrm{p} 53 \gamma$-mediated SMAD promoter activation (Fig. 4c and d). Together, the observations suggest that ectopic $\Delta 133 p 53 \gamma$ activates the SMAD promoter that leads to the restriction of cell migration (Fig. 4e).

\section{The WWOX/TIAF1/p53 triad induces apoptosis in cancer cells}

Next, we investigated whether the overexpressed WWOX/ TIAF1/p53 triad limits cell migration is due, in part, to 


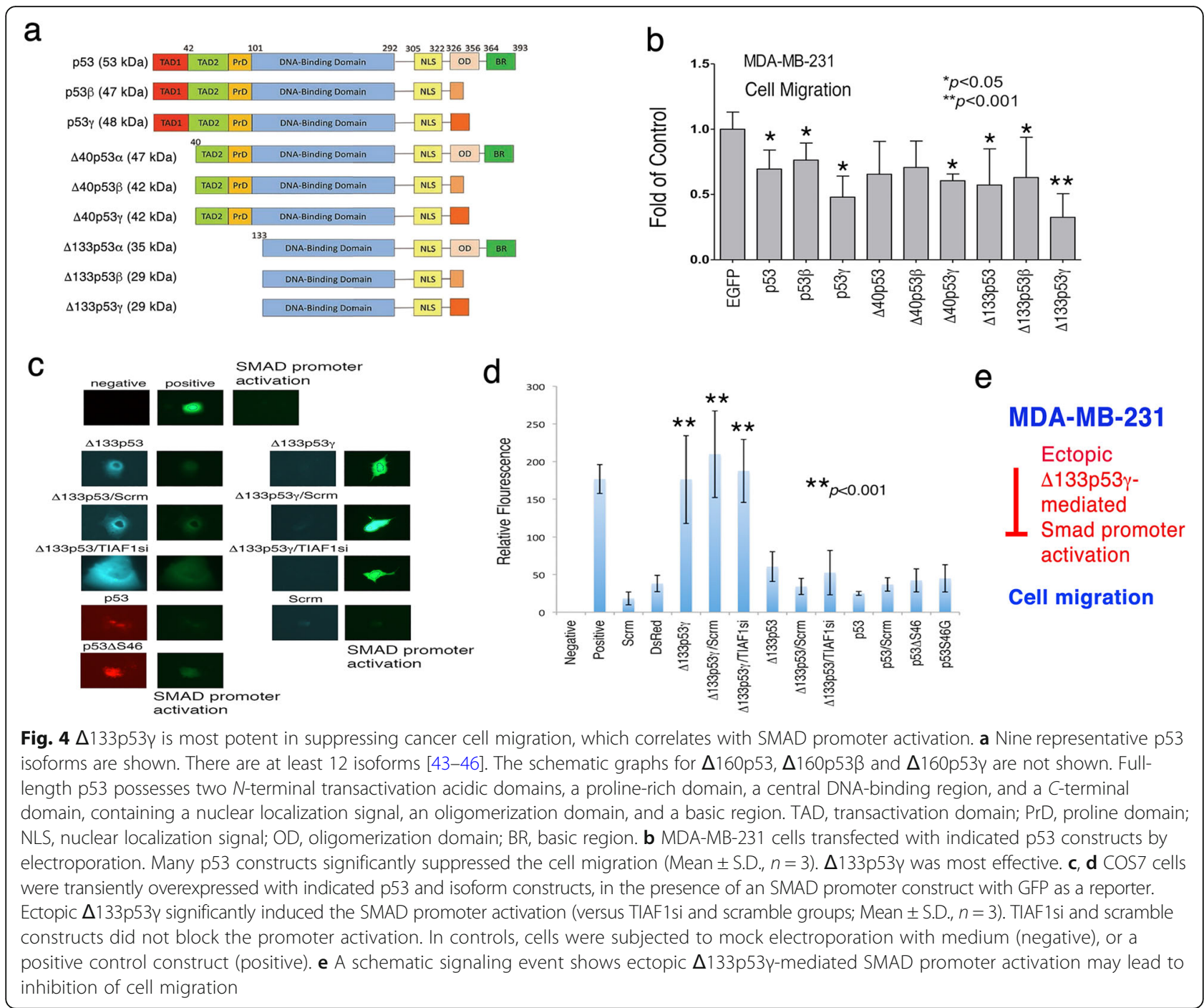

induced apoptosis. In agreement with our previous observations [23], ectopic expression of the triad proteins induced apoptosis of L929 cells (Fig. 5a). Similar results were observed using other types of cells such as breast MDAMB-231, COS7 fibroblasts, and lung NCI-H1299 cells. We have shown that pY33-WWOX physically binds pS46-p53, and the complex induces apoptosis [31]. More than $50 \%$ of cancer cells have p53 mutations and Ser46 is one of them [31, 43]. p53(S46G), which has an alteration of Ser46 to Gly, blocked the WWOX/TIAF1 complex-mediated apoptosis of L929 cells (see the last two columns to the right; Fig. 5a). However, p53(S46G) could not significantly suppress apoptosis induced by WWOX or TIAF1 alone (Fig. 5a). p53(S46G) does not bind WWOX [31].

\section{TIAF1 binds the first N-terminal WW domain of WWOX} We investigated how WWOX binds TIAF1. By Ras rescue-based Cytotrap yeast two-hybrid analysis [27, 29,
30, 34, 35, 38, 39], we determined the binding of TIAF1 to the $\mathrm{N}$-terminal first WW domain of WWOX, as revealed by mutant yeast growth at $37^{\circ} \mathrm{C}$ using a selective medium in agarose plates (Fig. 5b). When the conserved phosphorylation site Tyr33 is altered to Arg, WWOX(Y33R) could not bind TIAF1, suggesting that Tyr33 phosphorylation is involved. Also, the SDR domain of WWOX did not bind TIAF1. MafB self-association and WWOX/p53 binding showed the positive binding interactions (Fig. 5b). Empty vector pSos and empty vector pMyr did not yield in positive binding (Fig. 5b).

To further validate the TIAF1/WWOX binding, L929 cells were stimulated with TNF $\alpha$ for $30 \mathrm{~min}$. TNF $\alpha$ rapidly increased TIAF1 protein expression (Fig. 5c). Binding of TIAF1 with WWOX was observed by co-immunoprecipitation. Also, endogenous TIAF1 binds Tyr33-phosphorylated WWOX (pY33-WWOX) in breast MCF7 cells, and that TGF- $\beta 1$ had little or no effect on the 
a p53(S46G) abolishes WWOX/TIAF1-mediated apoptosis

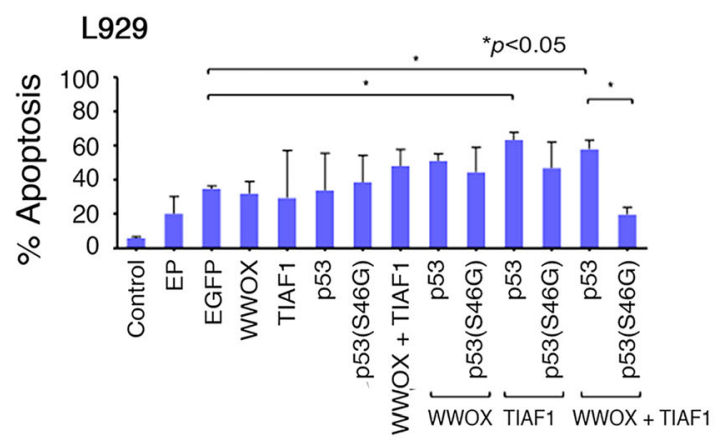

b TIAF1 binds the first WW domain of WWOX

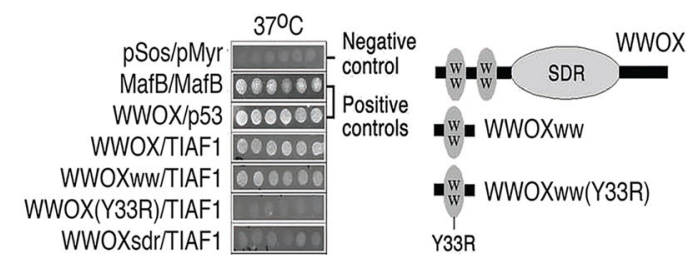

C TIAF1 binds WWOX

d pY33-WWOX binds TIAF1

\section{L929}

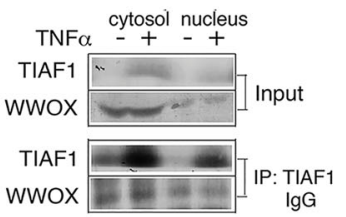

\section{MCF7}

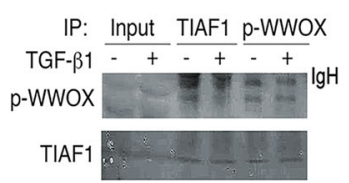

e Prima 1 increases WWOX/TIAF1/p53 complex formation

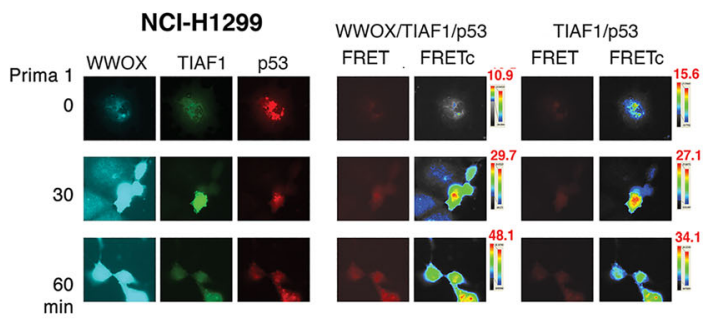

f Prima 1 increases complex formation

cos7

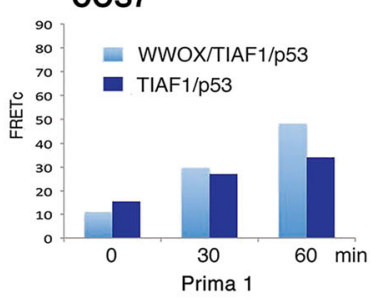

9 TGF- $\beta 1$ rapidly increases complex formation

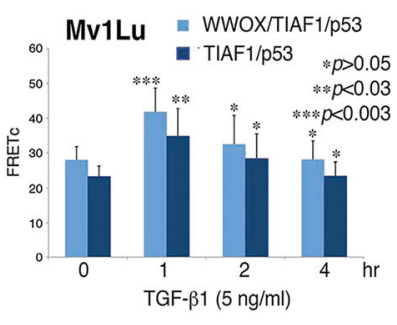

h The first WW domain binds TIAF1 and p53

\section{$\cos 7$}

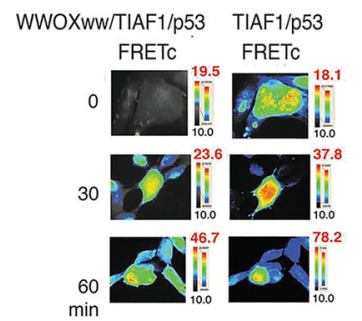

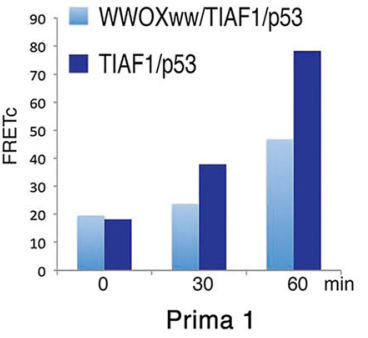

Fig. 5 Transiently overexpressed WWOX/TIAF1/p53 triad induces apoptosis, and determination of the kinetics of the triad formation. a L929 cells were electroporated with indicated plasmids. Overexpression of WWOX, TIAF1 and p53 induced apoptosis. Dominant-negative p53 (S46G) suppressed apoptosis mediated by p53 and TIAF1. EP: electroporation with medium only (Mean \pm S.D., $n=3,{ }^{*} p<0.05$, student's $t$ test). b Ras rescue-based yeast two-hybrid analysis revealed the binding of TIAF1 to the N-terminal first WW domain of WWOX, as shown by the growth of yeast at $37^{\circ} \mathrm{C}$ [29-31]. WWOX(Y33R) mutant abolished the binding. The SDR domain failed to bind TIAF1. In positive binding controls, MafB selfassociation and WWOX/p53 binding are shown, and empty pSos versus empty pMyr as negative controls. CDNAs constructed in the pSos are shown in the left, and pMyr in the right. c TNFa, at $100 \mathrm{ng} / \mathrm{ml}$, rapidly upregulated the expression of TIAF1 in 30 min in L929 cells. Co-immunoprecipitation showed the binding of TIAF1 with WWOX. $\mathbf{d}$ By co-immunoprecipitation, TGF- $\beta 1$ ( $5 \mathrm{ng} / \mathrm{ml})$ had little or no effect on the binding of TIAF1 with pY-WWOX during treatment for $30 \mathrm{~min}$ in MCF7 cells. IgH: IgG heavy chain. e, f NCl-H1299 cells were transiently transfected with p53-DsRed, TIAF1-EGFP, and WWOX-ECFP. By 3-protein FRET analysis [37, 39], Prima-1 induced p53 activation-dependent binding with WWOX and TIAF1 with time (see the number in red under FRETc columns, e Experiments were designed to let the FRET energy transfer from WWOX-ECFP to TIAF1-EGFP, and finally to p53-DsRed $[37,39]$. Prima- 1 increased the triad complex formation in COS7 cells (f). $\mathbf{g}$ In Mv1Lu cells, TGF- $\beta 1$ rapidly increased the triad complex formation in $1 \mathrm{~h}$, followed by reduction ( $n=6$; Student's $t$ test; experiments versus time 0 controls). $\mathbf{h}$ Prima- 1 increased the binding of the first WW domain of WWOX with TIAF1 and p53 with time. Each bar represents an average of two experiments in (f) and (h)

binding during treatment for $30 \mathrm{~min}$. (Fig. $5 \mathrm{~d}$ ). This data supports the observation from yeast two-hybrid analysis that pY33-WWOX binds TIAF1.

\section{Activated p53 binds TIAF1, and WWOX strengthens the p53/TIAF1 complex to stabilize the triad}

Under physiologic conditions, TIAF1 does not bind p53 [40]. However, under stress conditions, both p53 and WWOX are activated. pS46-p53 physically binds
pY33-WWOX and the complex translocates to the mitochondria or nucleus to induce apoptosis [29-31]. Here, p53-deficient NCI-H1299 cells were transiently transfected with p53-DsRed, TIAF1-EGFP, and WWOX-ECFP. Twenty-four hour later the cells were treated with Prima-1 for activating p53 [52]. By tri-molecular binding FRET analysis [37, 39], we measured the signal flow or the binding energy flow from WWOX-ECFP to TIAF1EGFP and then to p53-DsRed [37]. Prima-1 increased the 
binding of p53 with TIAF1 and WWOX in a time-related manner (Fig. 5e; see the number in red under FRETc columns). Interestingly, the binding strength of the whole triad was stronger than that of the activated p53/TIAF1 complex. Similar results were observed by using p53-positive COS7 cells transfected with the aforementioned constructs and treated with Prima-1 (Fig. 5f). Similarly, TGF- $\beta 1$ rapidly induced the triad complex formation in $1 \mathrm{~h}$, followed by reduction in epithelial Mv1Lu cells (Fig. 5g). The Mv1Lu cell is sensitive to TGF- $\beta$-mediated growth suppression [53]. The binding strength of p53 and TIAF1 was relatively weak, compared to the overall protein binding strength in the triad.

Next, COS7 cells were transiently transfected with the first WW domain of WWOX (WWOXww), along with TIAF1 and p53. Again, Prima-1 induced the triad formation with time (Fig. 5h). Interestingly, the p53/TIAF1 complex had a greater binding strength than that of the overall binding strength in the triad complex. The observations suggest that activated p53 binds TIAF1, and WWOX joins the complex via its $C$-terminal SDR domain to stabilize the complex triad.

\section{p53 and TIAF1 block WWOX and Smad4-induced SMAD promoter activation}

Suppressing one of the proteins in the triad abolished its tumor suppressor function [23] (Figs. 4 and 6), suggesting a concerted teamwork among p53, WWOX and TIAF1 is needed for cancer suppression. In light of the dynamic binding among the triad proteins (Fig. 5), we examined the potential functional antagonism among the triad proteins. p53-deficient lung cancer NCI-H1299 cells were transiently overexpressed with TIAF1-ECFP, p53-DsRed, and/or WWOX-ECFP, in the presence of the SMAD promoter construct. We determined that WWOX induced the SMAD promoter activation, and that p53 and/or TIAF1 abolished the activation (Fig. 6a). In a positive control, Smad4 induced the promoter activation (Fig. 6a; see left panel). Under similar conditions, p53 blocked WWOX-induced SMAD promoter activation in L929 cells (Fig. 6b). Furthermore, ectopic Smad4-mediated SMAD promoter activation was abolished by TIAF1 (Fig. 6c), which is in agreement with our previous report [23]. p53 and p53 $\Delta$ S46 induced Smad4-dependent SMAD promoter activation (Fig. 6c). TIAF1 and p53 (or p53 $\Delta$ S46) blocked Smad4-mediated SMAD promoter activation, and p53 $\Delta$ S46 less effective (Fig. 6c). The entire molecular event is summarized (Fig. 6d). Together, our data showed that p53 and TIAF1 may functionally interrupt with WWOX in affecting SMAD-dependent promoter activation.
WWOX suppresses cancer inflammation-mediated spleen enlargement and p53 abolishes the event, and the p53/ WWOX antagonism-induced inflammation leading to brain protein aggregation

NCI-H1299 cells were transiently overexpressed with DsRed-p53 and/or EGFP-WWOX, or EGFP only (Fig. 7a), followed by inoculating the cells in subcutaneous sites of both flanks in nude mice. WWOX-NCIH1299 cells had a suppressed growth in vivo. However, p53 abolished the growth suppression (Fig. 7a). Mice were sacrificed on day 86. Spleen enlargement was shown in mice inoculated with cells-expressing EGFP, DsRed-p53, or EGFP-WWOX/DsRed-p53 (Fig. 7b), suggesting that cancer-induced inflammation occurred. In contrast, WWOX blocked cancer cell-induced inflammation, so that the spleen size was the smallest (Fig. 7b). Notably, protein aggregates, including pERK, BACE, APP, $A \beta$, and NFT, were found in the brain of mice inoculated with p53/WWOX-expressing cells (Fig. 7c). Gels were run under both reducing and non-reducing conditions. These proteins are associated with neurodegeneration such as Alzheimer's disease [22, 24-26]. Housekeeping protein $\alpha$-tubulin was also aggregated. Additional proteins became aggregated were AIF and neurofilament middle and high molecular weights (NF$\mathrm{M} / \mathrm{H}$ ) (Fig. 7c). We have recently reported the occurrence of neurodegeneration during the progression of melanoma in mice [26]. No protein aggregates were found in the mice inoculated with cells expressing EGFP, DsRed-p53, or EGFP-WWOX alone (Fig. 7c). APP was shown to be degraded. Similarly, upregulated BACE, APP degradation, and $A \beta$ formation were also observed in the mouse lung (Fig. 7d). Aggregated BACE, APP, A $\beta$, BECN-1 and $\alpha$ Synuclein were shown in the lung.

\section{Discussion}

In agreement with our recent report [54], WWOXdeficient cancer cells and Wwox gene knockout MEF cells have loose intercellular contacts and they migrate individually. Also, their migration speed is much faster than that of the wild type cells. This accounts for the aggressive behavior of metastatic cancer cells [14]. The JAK2/STAT3 axis has been implicated in the enhanced breast cancer cell metastasis [14]. In parallel, a recent study showed that WWOX restricts the migration of triple negative breast cancer cells via regulating the expression of miR-146a [55]. We showed that aberrant signal pathways occur in the Wwox knockout MEF cells. These cells possess constitutive activation of Smad3 and p38, along with significant downregulation of p53, IкB $\alpha$, Smad6 and Fas. Whether these events contribute to enhanced cell proliferation of Wwox knockout MEF cells remains to be established. Indeed, the knockout cells 


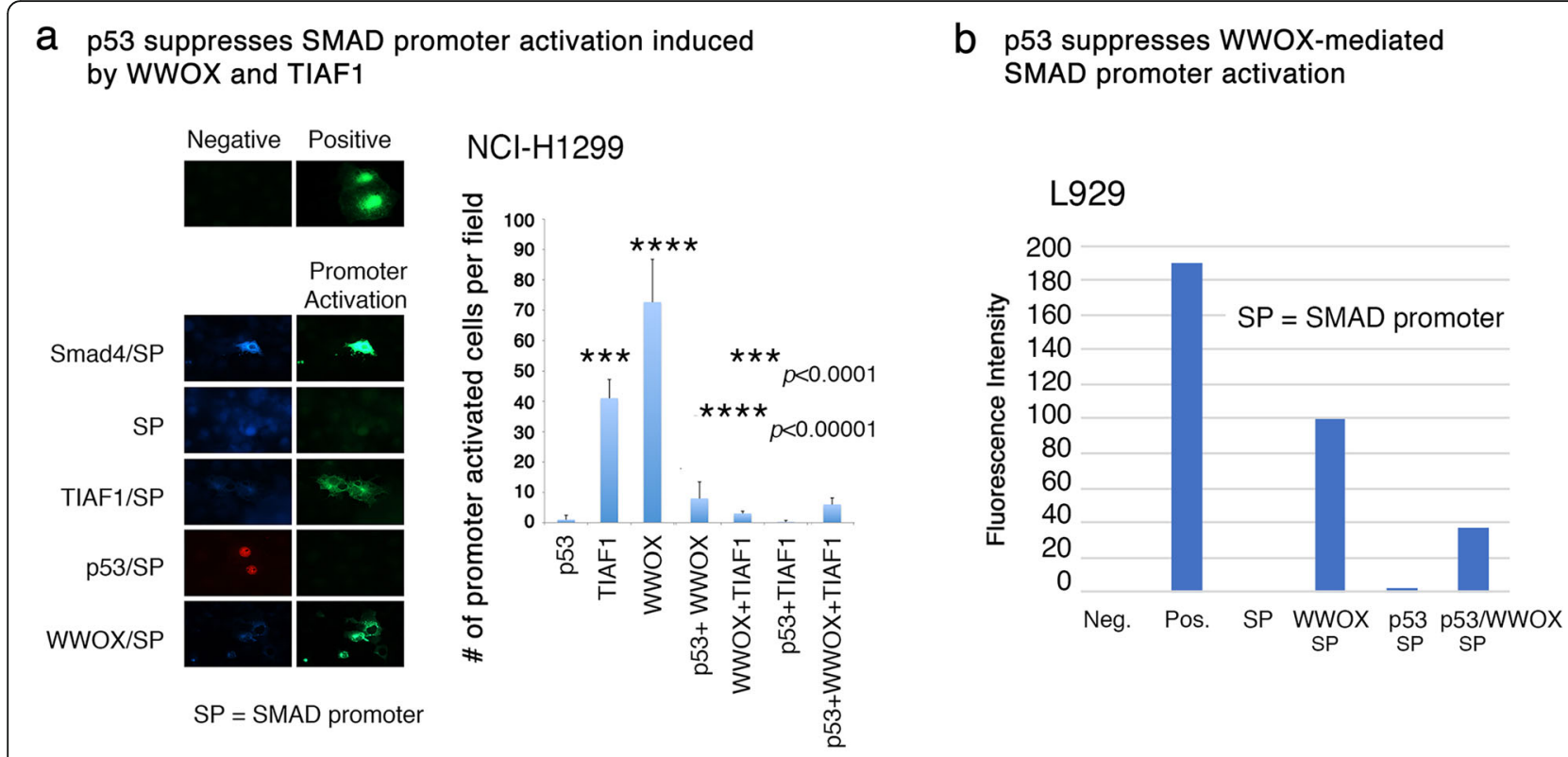

C Smad4-induced SMAD promoter activation is blocked by TIAF1

d Smad promoter (SP) activation
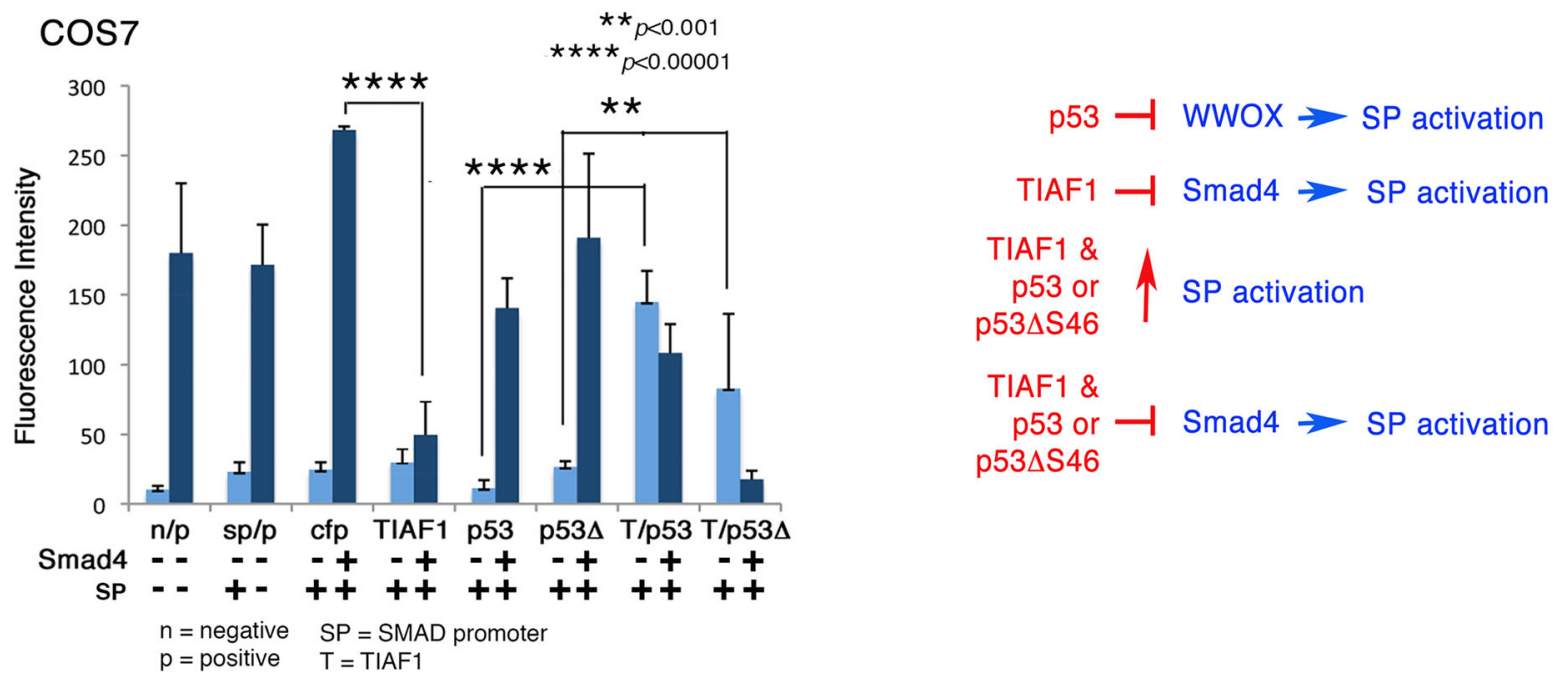

Fig. 6 p53 abolishes WWOX-induced SMAD promoter activation. a NCI-H1299 cells were transiently overexpressed with TIAF1-ECFP, p53-DsRed, and/or WWOX-ECFP in the presence of a SMAD promoter plasmid with EGFP as a reporter. WWOX and TIAF1 induced the SMAD promoter activation, and that p53 abolished the activation. In a positive control, Smad4 induced the promoter activation. $\mathbf{b}$ In parallel, WWOX-induced Samd4 promoter activation was blocked by p53 in L929 cells. c Similarly, COS7 cells were transfected with Smad4-ECFP and the SMAD promoter construct, in the presence or absence of p53, p53 $\Delta$ S46, and/or TIAF1. $\mathbf{d}$ In summary, data shows 1) p53 blocks WWOX-mediated SMAD promoter activation, 2) TIAF1 blocks Smad4-mediated SMAD promoter activation, 3) p53 or p53 $\Delta$ S46 increases Smad4-mediated SMAD promoter activation, and 4) TIAF1 blocks p53 or p53 $\triangle$ S46-increased Smad4-mediated SMAD promoter activation. SP = SMAD promoter

tend to undergo apoptosis post rapid proliferation (data not shown).

The wild type cells have strong cell-cell contacts and migrate collectively. WWOX undergoes Tyr33 phosphorylation and nuclear translocation upon stimulating cells with TGF- $\beta$ [34]. TGF- $\beta$ accelerates the collective migration of wild type MEF cells. Also, there is an increased cell number migrating individually, supporting the role of TGF- $\beta$ in promoting cancer cell migration and metastasis $[34,48]$. By co-culturing the wild type and Wwox knockout MEF cells, the knockout cells always undergo retrograde migration upon facing the wild type cells [54]. Multiple signal pathways, including MIF, Hyal-2, Eph, and Wnt pathways that converge to ERK signaling is responsible for the retrograde migration [54]. 


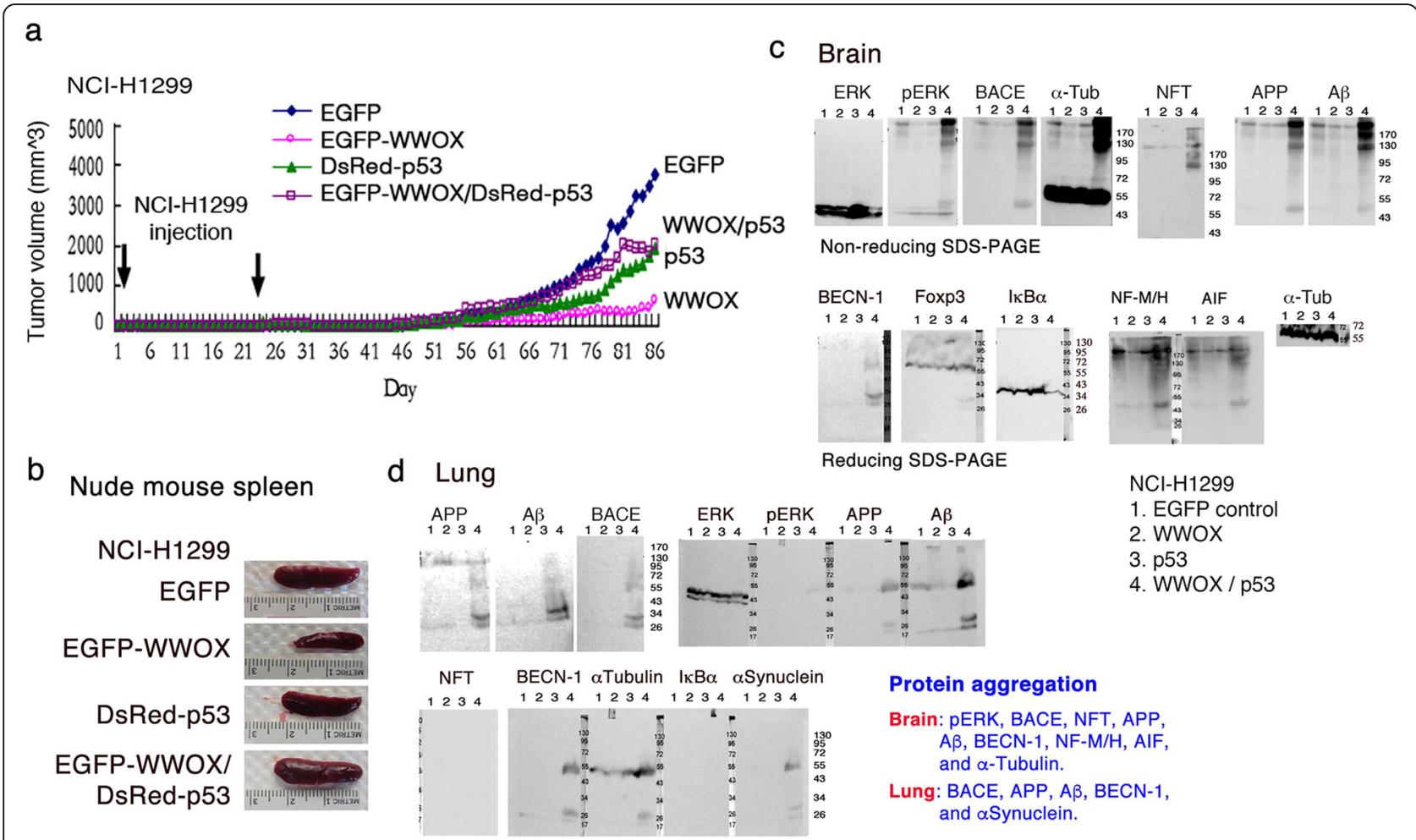

Fig. 7 p53 counteracts WWOX-mediated suppression of NCI-H1299 cell growth in nude mice and this antagonism leads to neurodegeneraiton in vivo. a NCI-H1299 cells were transiently overexpressed with p53-DsRed and/or WWOX-ECFP, or EGFP only. Nude mice received subcutaneous injections of these cells twice on both sides of the flanks. Tumor sizes were measured daily. WWOX suppressed tumor growth, and the suppression was abolished by p53. A representative data is shown from two experiments. b Mice were sacrificed on day 86 . Spleen enlargement occurred in all mice except in the mouse harboring WWOX-expressing NCl-H1299. c Protein aggregates were found for pERK, BACE, APP, AB, NFT and a-tubulin in the brain of mouse inoculated with p53/WWOX-expressing NCI-H1299 (top panel; nonreducing gels). APP was degraded. Under reducing conditions (bottom panel), aggregation of BECN-1, neurofilament NF-M/H, and AIF is shown. $\mathbf{d}$ Similar results were observed for the expression of BACE, APP, AB, $\mathrm{BECN}-1$, and aSynuclein in the mouse lung

We continued to show that the p53/TIAF1/WWOX axis is a protein triad for cancer suppression, including inhibition of cell migration, adherence-independent growth, and SMAD promoter activation, and induction of cancer cell apoptosis [23]. p53, TIAF1 and WWOX act in a concerted manner to inhibit cancer cell growth, migration and apoptosis in vitro. Missing a component in the p53/ TIAF1/WWOX triad reduces its cancer inhibitory function. WWOX, via its first $N$-terminal WW domain, physically binds p53 and TIAF1, suggesting that p53 and TIAF1 competitively bind WWOX. Under apoptotic stress, pY33-WWOX binds pS46-p53 to carry out apoptosis [31]. Binding of TIAF1 with WWOX depends upon phosphorylation of WWOX at Tyr33. TIAF1 undergoes phosphorylation Ser37 [22, 23, 28]. Whether pS37-TIAF1 binds pY33-WWOX is unknown. Like p53 and WWOX, TIAF1 is significantly downregulated or could be altered in many types of cancer cells $[23,28,56]$.

Under physiologic conditions, the binding strength for p53, WWOX and TIAF1 is weak. p53 fails to bind TIAF1 [53]. Intriguingly, Prima-1 activates p53, and this leads to the p53/TIAF1 complex formation. WWOX appears to further enhances the binding with p53/TIAF1 to increase the stability of the triad. The SDR domain of WWOX is probably involved in the triad stabilization. Whether TIAF1 is Ser37 phosphorylated in the triad remains to be determined. Both TNF $\alpha$ and TGF- $\beta 1$ rapidly increase the triad formation, further supporting the observations that the p53/TIAF1/WWOX triad participates in cancer growth suppression, migration inhibition, and apoptosis. Quite frequently, when two proteins bind reaching a maximal strength, the protein complex further drives to polymerization and leads to aggregation for causing apoptosis [22-24, 28]. Thus, rapid triad formation of p53, TIAF1 and WWOX is important for cancer suppression and causing cancer cell death. $\Delta 133 p 53 \gamma$ isoform strongly suppresses cancer cell migration, and this positively correlates with its-mediated SMAD promoter activation. We do not exclude the possibility that $\Delta 133 \mathrm{p} 53 \gamma$ undergoes self-association and this provides a driving energy to cause SMAD promoter activation and inhibition of cell migration. The molecular 
nature of the $\Delta 133 \mathrm{p} 53 \mathrm{y}$ isoform in cancer cells remains to be established.

p53 and WWOX are known to induce apoptosis in a synergistic manner [29-31]. We demonstrated for the first time that there is a functional antagonism between these two tumor suppressors. For example, p53 blocks WWOXinduced SMAD promoter activation. Notably, WWOXoverexpressing NCI-H1299 cells do not elicit inflammatory immune response. However, p53 abolishes the function of WWOX in causing cancer-mediated inflammation. This functional antagonism correlates with the increased tumor growth and neurodegeneration in vivo, as shown by growing p53/WWOX-expressing NCI-H1299 cells in nude mice. WWOX inhibition of NCI-H1299 tumor growth is blocked by p53. For unknown reasons, the growing tumors induce protein aggregation in the mouse brain and lung. We believe that cytokines released by growing cancer cells induce protein aggregation. These aggregated proteins include pERK, BACE, $\alpha$-tubulin, NFT, APP and A $\beta$, in which BACE upregulation, APP degradation, and A $\beta$ formation occur in the brain. BACE is responsible for cleaving APP that allows formation of $A \beta$ formation and plaque generation. The observations indicate there is an ongoing neurodegeneration in the brain of tumor-growing mice. We have recently shown that growing melanoma or glioblastoma in mice leads to neurodegeneration [26]. Downregulation of WWOX in the brain may lead to a cascade of protein aggregation, starting from TRAPPC6A $\triangle$, TIAF1, and SH3GLB2, which results in APP degradation, and aggregation of amyloid $\beta$ and tau [3, 23-26]. How the tumor cells in the flanks control neurodegeneration in the brain is unknown and remains to be established.

TIAF1 is a potential tumor suppressor. We demonstrated the anticancer function of TIAF1 by showing its critical role in cell death. TIAF1 rapid interaction with WWOX is essential in executing cell death. TIAF1 is upregulated in growing tumors, but may disappear in established metastatic cancer cells [23, 28]. TIAF1 protein aggregation has been shown in the human cortex and hippocampus of nondemented mid-aged humans and demented old patients [22, 24-26]. TIAF1 aggregates, together with Smad4 and $A \beta$, are found in the cancer stroma and peritumor capsules of many solid tumors [23]. Presence of TIAF1/A $\beta$ aggregates is shown on the interface between brain neural cells and the metastatic cancer cell mass. The TIAF1/A $\beta$ aggregates is toxic to neural cells but not cancer cells. Also, TIAF1 and amyloid fibrils are significantly accumulated in the stroma of progressing lung cancer cells [23]. These peritumor materials probably provide support for cancer cell survival.

TIAF1 undergoes self-association, which leads to increased expression of Smad4 and WWOX [23]. WWOX in turn increases the TIAF1 expression. Binding of TIAF1 with Smad4 induces $A \beta$ formation [23]. TIAF1 suppresses

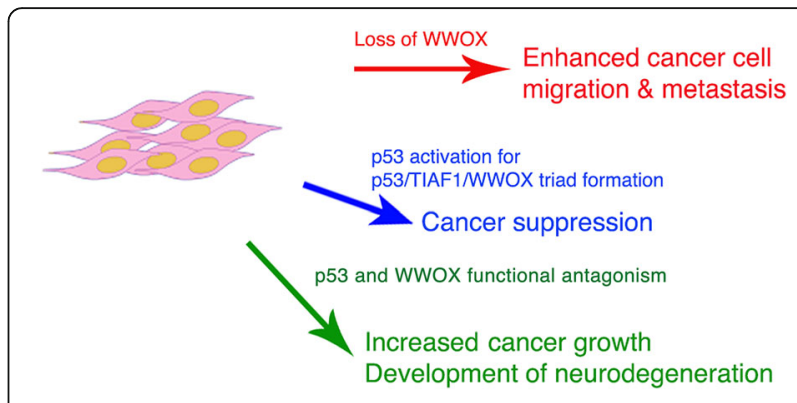

Fig. 8 Summary illustration. Three scenarios account for cancer growth or suppression: 1) loss of WWOX, p53 and TIAF1 increases cancer growth and metastasis; 2) stabilized p53/WWOX/TIAF1 triad suppresses cancer growth, inhibits metastasis, and induces

apoptosis; 3) functional antagonism between p53 and WWOX allows cancer cell growth and yet induces inflammation for causing neural protein aggregation as seen in the Alzheimer's disease

SMAD-regulated promoter activation. Notably, in the absence of p53, self-aggregating TIAF1 spontaneously activated the SMAD-regulated promoter. pS15-p53 requires the presence of TIAF1 to undergo nuclear translocation [40]. Together, our previous and current findings imply that Smad4 and p53 restrict TIAF1 self-aggregation, and that loss of tumor suppressors p53, WWOX, and Smad4 results in TIAF1 aggregate formation, which supports cancer growth and causes neurodegeneration.

\section{Conclusions}

In conclusion, we have provided strong evidence for the binding interactions among WWOX, TIAF1 and p53, and the firmly established WWOX/TIAF1/p53 protein triad exerts strong cancer suppression by blocking cancer cell migration, anchorage-independent growth and SMAD promoter activation, and inducing apoptosis (see Summary illustration, Fig. 8). Yet, p53 may functionally antagonize with WWOX. p53 blocks WWOX inhibition of inflammatory immune response induced by cancer, and this leads to protein aggregation in the brain as seen in the Alzheimer's disease and other neurodegeneration. pS14-WWOX is known to be associated with the severe progression of cancer and neurodegeneration $[1,26]$. Upregulation of pS14-WWOX in the lesions of cancer and neurodegeneration correlates with downregulation of pY33-WWOX. Apparently, pS14-WWOX favors inflammatory response for disease progression. Whether p53 binds pS14-WWOX to promote diseases is unknown.

\section{Additional files}

Additional file 1: Figure S1. Wild type MEF cells migrate collectively, whereas Wwox knockout MEF cells migrate individually. Shown is the imaging of cell migration at 0 and $48 \mathrm{~h}$ by time-lapse microscopy. Also, see Additional file 2: Video S1 and Additional file 3: Video S2. The image is digitally enlarged from Fig. 2d. Figure S2. WWOX-deficient cells have a 
faster migration rate. WWOX-deficient MDA-MB43 $5 \mathrm{~s}$ and MDA-MB-231 migrated faster than WWOX-positive L929 s cells. WWOX appears to be functionally deficient in MCF7 cells, as these cells migrated effectively compared to the WWOXnegative cells. This data links to Fig. 2. Figure S3. TGF- $\beta 1$ suppresses the migration of Wwox knockout cells. MEF cells were treated with TGF- $\beta 1$ or TGF- $\beta 2(10 \mathrm{ng} / \mathrm{ml})$ for $48 \mathrm{~h}$. Both TGF- $\beta 1$ and TGF- $\beta 2$ promoted wild type cell migration. TGF- $\beta 2$ is more effective in suppressing the knockout cell migration than TGF- $\beta 1$. This data links to Fig. 2i. Figure S4. TGF- $\beta 1$ does not affect the proliferation of Wwox MEF cells. TGF- $\beta 1$ had no significant effect on the cell proliferation of both wild type and the Wwox knockout MEF cells ( $n=3$, Student's $t$ test). This data links to Fig. 2. Figure S5. Colocalization of transiently overexpressed TIAF1 with p53 and WWOX proteins. MDA-MB-231 cells were transiently overexpressed with p53-DsRed, TIAF1-EGFP and WWOX-ECFP. TIAF1 underwent polymerization and retained p53 and WWOX in the cytoplasm (see punctate). The image data is enlarged from Fig. 3c. Figure S6. p53 is more potent than WWOX in blocking anchorage-independent cell growth. L929 cells were transfected with the following CDNA expression constructs for the anchorage-independent growth assay: 1) p53, 2) WWOX (OXFL), 3) p53 $3546(p 53 \Delta 46)$, 4) p53 MWOX, and 5) p53 $\Delta$ S46 MWOX. Data is shown as an average of duplicate experiments. This data supports Fig. $3 \mathrm{~d}$ and e. (PDF $8481 \mathrm{~kb}$ )

Additional file 2: Video S1. Migration of wild type $W_{W o x^{+/+}} \mathrm{MEF}$ cells as determined by time-lapse microscopy. Wild type WWox ${ }^{+/+}$MEF cells were cultured in the right and left chambers of a culture-insert (ibidi) for $24 \mathrm{~h}$. Following removal of the culture-insert, cells were allowed to migrate to each other from both sides. Time-lapse microscopy was performed at $37^{\circ} \mathrm{C}$ with 5\% CO2. (MP4 $\left.2361 \mathrm{~kb}\right)$

Additional file 3: Video S2. Migration of knockout Wwox $x^{-{ }^{-1}}$ MEF cells as determined by time-lapse microscopy. Knockout Wwox ${ }^{-1-}$ MEF cells were cultured in the right and left chambers of a culture-insert (ibidi) for $24 \mathrm{~h}$. Following removal of the culture-insert, cells were allowed to migrate to each other from both sides. Time-lapse microscopy was performed at $37^{\circ} \mathrm{C}$ with $5 \% \mathrm{CO} 2$. (MP4 $\left.3411 \mathrm{~kb}\right)$

\section{Abbreviations}

ADH/SDR: Short chain alcohol dehydrogenase/reductase; AlF: Apoptosisinducing factor; APP: Amyloid Precursor Protein; A $\beta$ : Amyloid beta; BACE: Betasecretase 1; BECN-1: Beclin-1; DMEM: Dulbecco's modified eagle medium; FBS: Fetal bovine serum; Foxp3: Forkhead box P3; Hyal-2: Hyaluronidase type 2; IKBa: Inhibitor of nuclear factor kappaB alpha; MEF: Mouse embryonic fibroblast; NF-M/H: Neurofilament middle and high molecular weights ( $M$ and $H$ ); NFT: Neurofibrillary tangles; NLS: Nuclear localization signal; NOS2: Nitric oxide synthase 2; NSYK motif: Asparagine-serine-tyrosine-lysine motif; PBS: Phosphate buffer saline; pERK: Phosphorylated extracellular signal-regulated kinases; SCC: Skin squamous cell carcinoma; SH3GLB2: SH3 Domain Containing GRB2 Like, Endophilin B2; TGF-B1: Transforming growth factor-B1; TIAF1: TGF-B1induced antiapoptotic factor; TRAPPC6A $\triangle$ : Trafficking protein particle complex 6A delta; WWOX or WOX1: WW domain-containing oxidoreductase

\section{Acknowledgements}

We appreciate Johnny Wen and Tom Lieu for managing the references.

\section{Authors' contributions}

P-YC carried out cell migration assays, time-lapse microscopy, apoptosis assay, gene cloning, transforming growth on agarose, and immunofluorescence microscopy, and wrote thesis for her Master degree as part of this manuscript. S-RL and M-HL performed gene cloning, plasmid purification, apoptosis assay, and transforming growth on agarose. LS carried out transforming growth on agarose. C-IS proofread the manuscript. N-SC conceived ideas, carried out promoter activation assay, FRET microscopy, co-immunoprecipitation, and yeast two-hybrid analysis, and wrote and revised the manuscript for submission. All authors read and approved the final manuscript.

\section{Authors' information}

Not applicable.

\section{Funding}

Research grants were funded to N-SC from the Ministry of Science and Technology, Taiwan (MOST 105-2320-B-006-046, 105-2320-B-006-036, 106-2320-
B-006-061, 106-2320-B-006-017, 107-2320-B-006 -058 -MY3, 107-2320-B-006005), and the Department of Defense, United States (W81XWH-08-1-0682), and the National Health Research Institutes, Taiwan (NHRI-EX107-10734NI for $\mathrm{N}-\mathrm{SC})$.

\section{Availability of data and materials}

All data generated or analyzed during this study are included in this article and the supplementary material.

\section{Ethics approval and consent to participate}

All experiments involved in animal use have been approved by the Institutional Animal Care and Use Committee (IACUC) of the National Cheng Kung University College of Medicine.

\section{Consent for publication}

All authors approved for the publication.

\section{Competing interests}

The authors declare that the research was conducted in the absence of any commercial or financial relationships that could be construed as a potential conflict of interest.

\section{Author details}

${ }^{1}$ Institute of Molecular Medicine, National Cheng Kung University, College of Medicine, Tainan, Taiwan 70101, Republic of China. ${ }^{2}$ Laboratory of Molecular Immunology, Guthrie Research Institute, Sayre, PA 18840, USA. ${ }^{3}$ Department of Cell Biology and Anatomy, National Cheng Kung University, College of Medicine, Tainan, Taiwan 70101, Republic of China. ${ }^{4}$ Department of Neurochemistry, New York State Institute for Basic Research in Developmental Disabilities, Staten Island, NY 10314, USA. ${ }^{5}$ Graduate Institute of Biomedical Sciences, College of Medicine, China Medical University, Taichung 40402, Taiwan, Republic of China.

Received: 8 February 2019 Accepted: 4 June 2019 Published online: 17 July 2019

\section{References}

1. Huang SS, Chang NS. Phosphorylation/de-phosphorylation in specific sites of tumor suppressor WWOX and control of distinct biological events. Exp Biol Med. 2018;243:137-47.

2. Pospiech K, Płuciennik E, Bednarek AK. WWOX tumor suppressor gene in breast Cancer, a historical perspective and future directions. Front Oncol. 2018;8:345.

3. Liu CC, Ho PC, Lee IT, Chen YA, Chu CH, Teng CC, Wu SN, Sze Cl, Chiang MF, Chang NS. WWOX phosphorylation, signaling, and role in neurodegeneration. Front Neurosci. 2018:12:563.

4. Tanna M, Aqeilan Rl. Modeling WWOX loss of function in vivo: what have we learned? Front Oncol. 2018;8:420.

5. Abu-Remaileh M, Dodson EJ, Schueler-Furman O, Aqeilan Rl. Pleiotropic functions of tumor suppressor WWOX in normal and cancer cells. J Biol Chem. 2015;290:30728-35.

6. Chang NS, Hsu LJ, Lin YS, Lai FJ, Sheu HM. WW domain-containing oxidoreductase: a candidate tumor suppressor. Trends Mol Med. 2007; 13:12-22

7. Bednarek AK, Laflin KJ, Daniel RL, Liao Q, Hawkins KA, Aldaz CM. WWOX, a novel WW domain-containing protein mapping to human chromosome 16q23. 3-24.1, a region frequently affected in breast cancer. Cancer Res. 2000;60:2140-5.

8. Ried K, Finnis M, Hobson L, Mangelsdorf M, Dayan S, Nancarrow JK, Woollatt E, Kremmidiotis G, Gardner A, Venter D, Baker E, Richards RI. Common chromosomal fragile site FRA16D sequence: identification of the FOR gene spanning FRA16D and homozygous deletions and translocation breakpoints in cancer cells. Hum Mol Genet. 2000;9:1651-63.

9. He D, Zhang YW, Zhang NN, Zhou L, Chen JN, Jiang Y, Shao CK. Aberrant gene promoter methylation of p16, FHIT, CRBP1, WWOX, and DLC-1 in Epstein-Barr virus-associated gastric carcinomas. Med Oncol. 2015;32:92.

10. Yan H, Sun J. Methylation status of WWOX gene promoter CpG islands in epithelial ovarian cancer and its clinical significance. Biomed Rep. 2013;1:375-8 
11. Yan J, Zhang M, Zhang J, Chen X, Zhang X. Helicobacter pylori infection promotes methylation of WWOX gene in human gastric cancer. Biochem Biophys Res Commun. 2011;408:99-102.

12. Baykara O, Demirkaya A, Kaynak K, Tanju S, Toker A, Buyru N. WWOX gene may contribute to progression of non-small-cell lung cancer (NSCLC). Tumor Biol. 2010;31:315-20.

13. Lai FJ, Cheng CL, Chen ST, Wu CH, Hsu LJ, Lee JY, Chao SC, Sheen MC, Shen CL, Chang NS, Sheu HM. WOX1 is essential for UVB irradiation-induced apoptosis and Down-regulated via translational blockade in UVB-induced cutaneous squamous cell carcinoma in vivo. Clin Cancer Res. 2005;11:5769-77.

14. Chang R, Song L, Xu Y, Wu Y, Dai C, Wang X, Sun X, Hou Y, Li W, Zhan X. Loss of Wwox drives metastasis in triple-negative breast cancer by JAK2/ STAT3 axis. Nat Commun. 2018;9:3486.

15. Gourley C, Paige AJ, Taylor KJ, Ward C, Kuske B, Zhang J, Sun M, Janczar S, Harrison DJ, Muir M. WWOX gene expression abolishes ovarian cancer tumorigenicity in vivo and decreases attachment to fibronectin via integrin a3. Cancer Res. 2009;69:4835-42.

16. Zheng Q, Zhou Y, You Q, Shou F, Pang Q, Chen J. WWOX inhibits the invasion of lung cancer cells by downregulating RUNX2. Cancer Gene Ther. 2016;23:433

17. Del Mare S, Aqeilan RI. Tumor suppressor WWOX inhibits osteosarcoma metastasis by modulating RUNX2 function. Sci Rep. 2015;5:12959.

18. Kunkle BW, Grenier-Boley B, Sims R, Bis JC, Damotte V, Naj AC, Boland A, Vronskaya M, van der Lee SJ, Amlie-Wolf A, et al. Genetic meta-analysis of diagnosed Alzheimer's disease identifies new risk loci and implicates $A \beta$, tau, immunity and lipid processing. Nat Genet. 2019;51:414-30.

19. Aldaz CM, Ferguson BW, Abba MC. WWOX at the crossroads of cancer, metabolic syndrome related traits and CNS pathologies. Biochim Biophys Acta Rev Cancer. 2014;1846:188-200.

20. Abdel-Salam G, Thoenes M, Afifi HH, Körber F, Swan D, Bolz HJ. The supposed tumor suppressor gene WWOX is mutated in an early lethal microcephaly syndrome with epilepsy, growth retardation and retinal degeneration. Orphanet J Rare Dis. 2014;9:12.

21. Chen ST, Chuang J, Wang J, Tsai M, Li H, Chang NS. Expression of WW domain-containing oxidoreductase WOX 1 in the developing murine nervous system. Neuroscience. 2004;124:831-9.

22. Lee MH, Lin SR, Chang JY, Schultz L, Heath J, Hsu LJ, Kuo YM, Hong Q, Chiang MF, Gong CX, Sze Cl, Chang NS. TGF- $\beta$ induces TIAF1 selfaggregation via type $\|$ receptor-independent signaling that leads to generation of amyloid $\beta$ plaques in Alzheimer's disease. Cell Death Dis. 2010;1:e110

23. Chang JY, Chiang MF, Lin SR, Lee MH, He H, Chou PY, Chen SJ, Chen YA, Yang LY, Lai FJ, Hsieh CC, Hsieh TH, Sheu HM, Sze Cl, Chang NS. TIAF1 selfaggregation in peritumor capsule formation, spontaneous activation of SMAD-responsive promoter in p53-deficient environment, and cell death. Cell Death Dis. 2012;3:e302.

24. Chang J, Chang N. WWOX dysfunction induces sequential aggregation of TRAPPC6A $\triangle$, TIAF1, tau and amyloid $\beta$, and causes apoptosis. Cell Death Discov. 2015;1:15003.

25. Chang JY, Lee MH, Lin SR, Yang LY, Sun HS, Sze Cl, Hong Q, Lin YS, Chou YT, Hsu LJ, Jan MS, Gong CX, Chang NS. Trafficking protein particle complex $6 \mathrm{~A}$ delta (TRAPPC6A $\triangle$ ) is an extracellular plaque-forming protein in the brain. Oncotarget. 2015:6:3578.

26. Lee MH, Shih YH, Lin SR, Chang JY, Lin YH, Sze Cl, Kuo YM, Chang NS. Zfra restores memory deficits in Alzheimer's disease triple-transgenic mice by blocking aggregation of TRAPPC6A $\triangle$, SH3GLB2, tau, and amyloid $\beta$, and inflammatory NF-KB activation. Alzheimers Dement (N Y). 2017:3:189-204.

27. Mohamoud HS, Ahmed S, Jelani M, Alrayes N, Childs K, Vadgama N, Almramhi MM, Al-Aama JY, Goodbourn S, Nasir J. A missense mutation in TRAPPC6A leads to build-up of the protein, in patients with a neurodevelopmental syndrome and dysmorphic features. Sci Rep. 2018:8:2053.

28. Hong Q, Hsu L, Chou PY, Chou YT, Lu CY, Chen YA, Chang NS. Selfaggregating TIAF1 in lung cancer progression. Transl Respir Med. 2013;1:5.

29. Chang NS, Pratt N, Heath J, Schultz L, Sleve D, Carey GB, Zevotek N. Hyaluronidase induction of a WW domain-containing oxidoreductase that enhances tumor necrosis factor cytotoxicity. J Biol Chem. 2001;276:3361-70.

30. Chang NS, Doherty J, Ensign A. JNK1 physically interacts with WW domaincontaining oxidoreductase (WOX1) and inhibits WOX1-mediated apoptosis. J Biol Chem. 2003;278:9195-202.

31. Chang NS, Doherty J, Ensign A, Schultz L, Hsu LJ, Hong Q. WOX1 is essential for tumor necrosis factor-, UV light-, staurosporine-, and p53-mediated cell death, and its tyrosine 33-phosphorylated form binds and stabilizes serine 46-phosphorylated p53. J Biol Chem. 2005;280:43100-8.

32. Hu BS, Tan JW, Zhu GH, Wang DF, Zhou X, Sun ZQ. WWOX induces apoptosis and inhibits proliferation of human hepatoma cell line SMMC7721. World J Gastroenterol. 2012;18:3020.

33. Bonin F, Taouis K, Azorin P, Petitalot A, Tariq Z, Nola S, Bouteille N, Tury S, Vacher S, Bièche I. VOPP1 promotes breast tumorigenesis by interacting with the tumor suppressor WWOX. BMC Biol. 2018;16:109.

34. Hsu LJ, Schultz L, Hong Q, Van Moer K, Heath J, Li MY, Lai FJ, Lin SR, Lee $\mathrm{MH}$, Lo CP, Lin YS, Chen ST, Chang NS. Transforming growth factor $\beta 1$ signaling via interaction with cell surface Hyal-2 and recruitment of WWOX/ WOX1. J Biol Chem. 2009;284:16049-59.

35. Hsu LJ, Hong Q, Chen ST, Kuo HL, Schultz L, Heath J, Lin SR, Lee MH, Li DZ, Li ZL, Cheng HC, Armand G, Chang NS. Hyaluronan activates Hyal-2/WWOX/ Smad4 signaling and causes bubbling cell death when the signaling complex is overexpressed. Oncotarget. 2017:8:19137.

36. Chen SJ, Lin PW, Lin HP, Huang SS, Lai FJ, Sheu HM, Hsu LJ, Chang NS. UV irradiation/cold shock-mediated apoptosis is switched to bubbling cell death at low temperatures. Oncotarget. 2015;6:8007.

37. Kuo HL, Ho PC, Huang SS, Chang NS. Chasing the signaling run by trimolecular time-lapse FRET microscopy. Cell Death Discov. 2018:4:45.

38. Chang NS, Schultz L, Hsu L, Lewis J, Su M, Sze Cl. 17ß-estradiol upregulates and activates WOX1/WWOXV1 and WOX2MWOXV2 in vitro: potential role in cancerous progression of breast and prostate to a premetastatic state in vivo. Oncogene. 2005;24:714.

39. Huang SS, Su WP, Lin HP, Kuo HL, Wei HL, Chang NS. Role of WW domaincontaining oxidoreductase WWOX in driving T cell acute lymphoblastic leukemia maturation. J Biol Chem. 2016;291:17319-31.

40. Schultz L, Khera S, Sleve D, Heath J, Chang NS. TIAF1 and p53 functionally interact in mediating apoptosis and silencing of TIAF1 abolishes nuclear translocation of serine 15-phosphorylated p53. DNA Cell Biol. 2004:23:67-74.

41. Chang NS, Khera S. TIAF1 participates in the transforming growth factor beta1--mediated growth regulation. Ann N Y Acad Sci. 2003;995:11-21.

42. Chang NS, Mattison J, Cao H, Pratt N, Zhao Y, Lee C. Cloning and characterization of a novel transforming growth factor-beta1-induced TIAF1 protein that inhibits tumor necrosis factor cytotoxicity. Biochem Biophys Res Commun. 1998;253:743-9.

43. Muller PA, Vousden KH, Norman JC. p53 and its mutants in tumor cell migration and invasion. J Cell Biol. 2011;192:209-18.

44. Joruiz SM, Bourdon JC. p53 isoforms: key regulators of the cell fate decision. Cold Spring Harb Perspect Med. 2016;6(8):1-21..

45. Vieler M, Sanyal S. p53 Isoforms and their implications in cancer. Cancers (Basel). 2018;10:288. https://doi.org/10.3390/cancers10090288.

46. Kazantseva M, Mehta S, Eiholzer RA, Hung N, Wiles A, Slatter TL, Braithwaite AW. A mouse model of the $\Delta 133 p 53$ isoform: roles in cancer progression and inflammation. Mamm Genome. 2018:29:831-42.

47. Sze Cl, Su M, Pugazhenthi S, Jambal P, Hsu LJ, Heath J, Schultz L, Chang NS. Down-regulation of WW domain-containing oxidoreductase induces tau phosphorylation in vitro. A potential role in Alzheimer's disease. J Biol Chem. 2004;279:30498-506.

48. Soleimani A, Pashirzad M, Avan A, Ferns GA, Khazaei M, Hassanian SM Role of the transforming growth factor- $\beta$ signaling pathway in the pathogenesis of colorectal cancer. J Cell Biochem. 2018. https://doi.org/ 10.1002/jcb.28331 Review.

49. Ferraiuolo M, Verduci L, Blandino G, Strano S. Mutant p53 Protein and the Hippo Transducers YAP and TAZ: A Critical Oncogenic Node in Human Cancers. Int J Mol Sci. 2017;18. https://doi.org/10.3390/ijms18050961 Review.

50. Mello SS, Attardi LD. Deciphering p53 signaling in tumor suppression. Curr Opin Cell Biol. 2018;51:65-72.

51. Gadea G, de Toledo M, Anguille C, Roux P. Loss of p53 promotes RhoAROCK-dependent cell migration and invasion in 3D matrices. J Cell Biol. 2007;178:23-30.

52. Bykov VJ, Issaeva N, Shilov A, Hultcrantz M, Pugacheva E, Chumakov $P$, Bergman J, Wiman KG, Selivanova G. Restoration of the tumor suppressor function to mutant p53 by a low-molecular-weight compound. Nat Med. 2002;8:282-8

53. Chang NS. The non-ankyrin C terminus of Ikappa Balpha physically interacts with p53 in vivo and dissociates in response to apoptotic stress, hypoxia, DNA damage, and transforming growth factor-beta 1-mediated growth suppression. J Biol Chem. 2002;277:10323-31. 
54. Chou PY, Lai FJ, Chen YA, Sie YD, Kuo HL, Su WP, Wu CY, Liu TY, Wen KY, LiJin Hsu LJ, Sze Cl, Chang NS. Strategies by which WWOX-deficient metastatic cancer cells utilize to survive via dodging, compromising, and causing damage to WWOX-positive normal microenvironment. Cell Death Discov. 2019;5:97.

55. Khawaled S, Suh SS, Abdeen SK, Monin J, Distefano R, Nigita G, Croce CM, Aqeilan RI. WWOX inhibits metastasis of triple-negative breast Cancer cells via modulation of miRNAs. Cancer Res. 2019;79:1784-98.

56. Xing J, Liu C. Identification of genes associated with histologic tumor grade of esophageal squamous cell carcinoma. FEBS Open Bio. 2017;7:1246-57.

\section{Publisher's Note}

Springer Nature remains neutral with regard to jurisdictional claims in published maps and institutional affiliations.

Ready to submit your research? Choose BMC and benefit from:

- fast, convenient online submission

- thorough peer review by experienced researchers in your field

- rapid publication on acceptance

- support for research data, including large and complex data types

- gold Open Access which fosters wider collaboration and increased citations

- maximum visibility for your research: over $100 \mathrm{M}$ website views per year

At BMC, research is always in progress.

Learn more biomedcentral.com/submissions 